\title{
Association of COVID-19 inflammation with activation of the C5a-C5aR1 axis
}

https://doi.org/10.1038/s41586-020-2600-6

Received: 29 April 2020

Accepted: 23 July 2020

Published online: 29 July 2020

Check for updates

\author{
Julien Carvelli ${ }^{1,2,12}$, Olivier Demaria ${ }^{3,12}$, Frédéric Vély ${ }^{4,5,12}$, Luciana Batista ${ }^{3}$, \\ Nassima Chouaki Benmansour ${ }^{6,7}$, Joanna Fares ${ }^{3}$, Sabrina Carpentier ${ }^{3}$, Marie-Laure Thibult ${ }^{3}$, \\ Ariane Morel ${ }^{3}$, Romain Remark ${ }^{3}$, Pascale André ${ }^{3}$, Agnès Represa ${ }^{3}$, Christelle Piperoglou ${ }^{4,5}$, \\ the Explore COVID-19 IPH group*, the Explore COVID-19 Marseille Immunopole group*, \\ Pierre Yves Cordier ${ }^{6}$, Erwan Le Dault ${ }^{6}$, Christophe Guervilly ${ }^{2,8}$, Pierre Simeone $^{2,9}$, \\ Marc Gainnier ${ }^{1,2}$, Yannis Morel $^{3}$, Mikael Ebbo ${ }^{4,10}$, Nicolas Schleinitz $^{4,10}$ \& Eric Vivier ${ }^{3,4,5 凶}$
}

Coronavirus disease 2019 (COVID-19) is a disease caused by infection with severe acute respiratory syndrome coronavirus 2 (SARS-CoV-2) and has resulted in a pandemic ${ }^{1}$. The C5a complement factor and its receptor C5aR1 (also known as CD88) have a key role in the initiation and maintenance of several inflammatory responses by recruiting and activating neutrophils and monocytes ${ }^{1}$. Here we provide a longitudinal analysis of immune responses, including phenotypic analyses of immune cells and assessments of the soluble factors that are present in the blood and bronchoalveolar lavage fluid of patients at various stages of COVID-19 severity, including those who were paucisymptomatic or had pneumonia or acute respiratory distress syndrome. The levels of soluble C5a were increased in proportion to the severity of COVID-19 and high expression levels of $\mathrm{C} 5 \mathrm{aR} 1$ receptors were found in blood and pulmonary myeloid cells, which supports a role for the C5a-C5aR1 axis in the pathophysiology of acute respiratory distress syndrome. Anti-C5aR1 therapeutic monoclonal antibodies prevented the C5a-mediated recruitment and activation of human myeloid cells, and inhibited acute lung injury in human $\mathrm{C} 5 \mathrm{aR} 1$ knock-in mice. These results suggest that blockade of the C5a-C5aR1 axis could be used to limit the infiltration of myeloid cells in damaged organs and prevent the excessive lung inflammation and endothelialitis that are associated with acute respiratory distress syndrome in patients with COVID-19.
Most patients with COVID-19 have only a few mild symptoms, but about $15 \%$ of patients progress to severe pneumonia, and about $5 \%$ develop acute respiratory distress syndrome (ARDS), for which effective therapeutic strategies are urgently required ${ }^{2}$. The immune system has a dual role in the pathology of COVID-19, contributing to both virus elimination and the development of $\mathrm{ARDS}^{2}$. A detailed characterization of the immune responses that occur during disease progression from mild to severe forms is therefore important for understanding the ways in which we can manipulate immunity to propose new therapies. In particular, given the urgent need for effective treatments for pneumonia in patients with COVID-19, the elucidation of the immune responses that occur during the course of COVID-19 could lead to the repurposing of approved immunomodulatory drugs and candidate drugs that have already been tested in clinical trials. We therefore monitored immune parameters in a cohort of 82 individuals: 10 healthy control individuals, 10 patients with COVID-19 who were paucisymptomatic, 34 patients with pneumonia and 28 patients with ARDS due to SARS-CoV- 2 infection
(Supplementary Table 1). We focused on molecular pathways that could block the overt inflammation associated with ARDS.

Disease severity was associated with an increase in the amounts of plasma C-reactive protein (CRP) and inflammatory cytokines-such as interleukin-6 (IL-6), and the chemokines CCL4 (macrophage inflammatory protein-1 $\beta$ ), CCL2 (monocyte chemoattractant protein 1 ) and CXCL9 (monokine induced by interferon- $\gamma$ ) - that are produced by and act on myeloid cells (Fig. 1a). These results confirmed previous observations of the 'cytokine storm' that develops in patients with severe COVID-193. The ability of plasma from patients to neutralize the SARS-CoV-2 virus was also correlated with disease severity (Extended Data Fig. 1a), consistent with previous studies, which show higher titres of anti-SARS-CoV-2 antibodies in patients with severe COVID-19 ${ }^{4}$.

We decided to focus on the complement factor C5a, which mediates strong chemoattraction and activation of myeloid cells ${ }^{5}$, and has well-documented roles specifically in lung inflammation and injury ${ }^{6}$. The complement cascade is important for the sensing and clearance

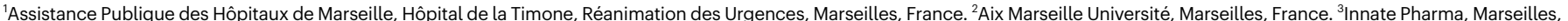
France. ${ }^{4}$ Aix Marseille Université, CNRS, INSERM, CIML, Marseilles, France. ${ }^{5}$ Assistance Publique des Hôpitaux de Marseille, Hôpital de la Timone, Immunology, Marseille Immunopole, Marseilles, France. ${ }^{6}$ Hôpital d'Instruction des Armées Laveran, Marseilles, France. ${ }^{7}$ Assistance Publique des Hôpitaux de Marseille, Marseilles, France. ${ }^{8}$ Assistance Publique des Hôpitaux de Marseille, Hôpital Nord, Réanimation des Détresses Respiratoires et Infections Sévères, Aix-Marseille Université, Marseilles, France. ${ }^{9}$ Assistance Publique des Hôpitaux de Marseille, Hôpital de la Timone, Réanimation Polyvalente, Aix-Marseille Université, Marseilles, France. ${ }^{10}$ Assistance Publique des Hôpitaux de Marseille, Hôpital de la Timone, Internal Medicine, Marseilles,

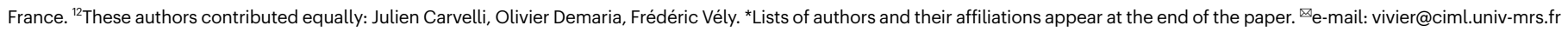



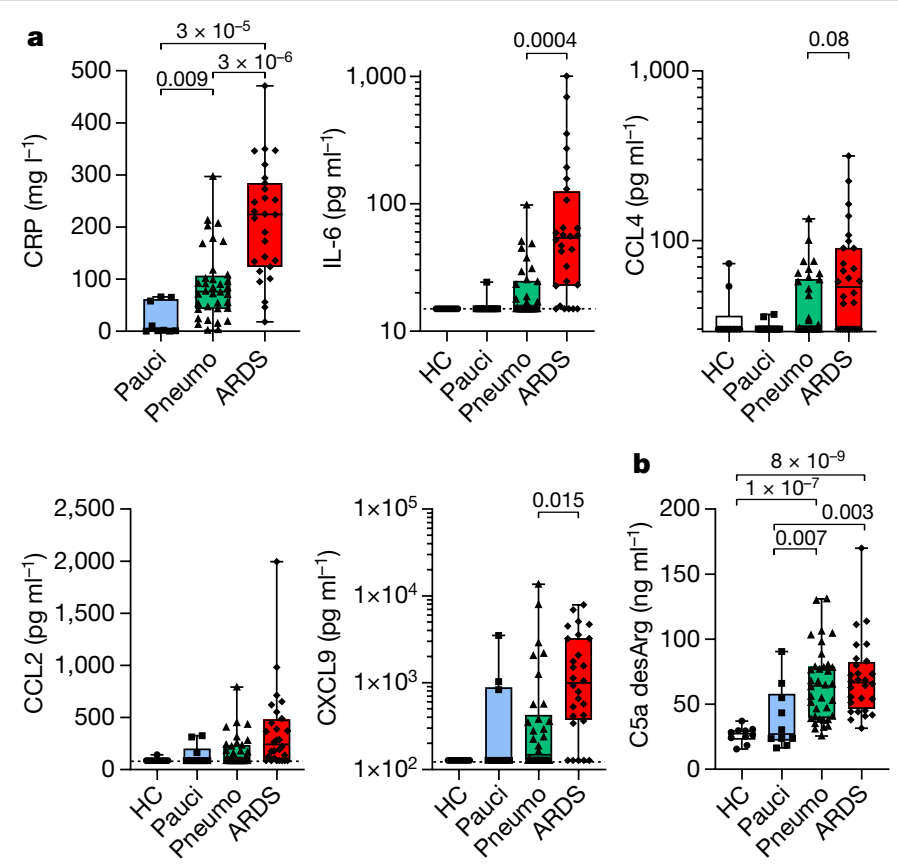

Fig. 1 | Inflammation is associated with a cytokine storm and C5a production in patients with COVID-19. a, Concentrations of CRP, IL-6, CCL4, CCL2 and CXCL9 in plasma from healthy control individuals (HC) and patients with COVID-19.b, Concentration of C5a desArg in plasma of $\mathrm{HC}$ and patients with COVID-19. Healthy control individuals, $n=10$; patients who were paucisymptomatic (pauci), $n=10$; patients with pneumonia (pneumo), $n=31-34$; patients with ARDS, $n=26-28$. For CRP $(\mathbf{a})$ and $\mathbf{b}, P$ values were computed using two-tailed Wilcoxon rank-sum tests. For IL-6, CCL4, CCL2 and CXCL9 (a), a global comparison was first performed in which all values were classified into two categories: above or below the limit of quantification ( $P$ values for two-sided Fisher's exact tests show that there is an increase in the number of values above the limit of quantification with increasing severity); Fisher tests: $P=5 \times 10^{-7}$ for IL- $6, P=0.02$ for CCL $4, P=0.001$ for CCL2, $P=9 \times 10^{-6}$ for CXCL9. A comparison was then performed between patients with pneumonia and with ARDS, only for values above the limit of quantification ( $P$ values were computed using two-tailed Wilcoxon rank-sum tests). Each symbol represents a single donor. Box plots show the median and the 25 th to 75th percentiles, and the whiskers denote the maximum and minimum values. The dotted lines for IL-6, CCL2 and CXCL9 (a) indicate the limit of quantification.

of pathogens and inflammation ${ }^{7}$, and involves several components, including cell surface receptors and soluble regulators. In the final phase of the response, the membrane attack complex (MAC: C5b9) and the potent chemoattractants and inflammatory mediators $\mathrm{C} 3 \mathrm{a}$ and $\mathrm{C} 5 \mathrm{a}$ are generated. The MAC forms transmembrane channels on the surface of pathogen cells, disrupting the cell membrane and leading to cell death. The $\mathrm{C} 3 \mathrm{a}$ and $\mathrm{C} 5 \mathrm{a}$ proteins regulate inflammation by binding to their respective receptors, $\mathrm{C} 3 \mathrm{aR}$ and $\mathrm{C} 5 \mathrm{aR} 1^{8}$. Exaggerated complement activation contributes to the pathogenesis of many inflammatory and immune diseases ${ }^{5}$. Numerous studies of the lung epithelium have reported depositions of complement components during inflammation and suggested that the systemic activation of complement leads, through $\mathrm{C} 5 \mathrm{a}$, to the recruitment, activation and adhesion of neutrophils to the pulmonary endothelium, which results in cell damage, and subsequent acute lung injury (ALI) and ARDS, which can be fatal ${ }^{1,6,9}$. We observed an increase in plasma C5a levels that was proportional to COVID-19 severity (Fig. 1b). C5a levels increased in a few patients in the paucisymptomatic group, and were significantly higher in individuals with lung damage in the pneumonia and ARDS groups than in healthy controls (Fig. 1b). The longitudinal follow-up of patients with COVID-19 revealed that the upregulation of circulating C5a levels was maintained for at least 10 days after the inclusion of the patients in our cohort (Extended Data Fig. 1b). The higher level of C5a in the patients with the most-severe symptoms suggests a role for this anaphylatoxin in the inflammation that occurs in patients who develop ARDS. Increased systemic and local complement pathway activity was confirmed by transcriptomic analysis of peripheral blood from patients with COVID-19, which showed an upregulation of $C 1 Q$ and $C 2$ expression (Extended Data Fig. 1c) and by the presence of C5b9, as shown by immunostaining, in lung sections from patients with COVID-19 (Extended Data Fig.1d). Consistent with these results, high levels of C5a in patients with COVID-19 have recently been reported to be a consequence of overt activation of the lectin pathway of the complement cascade by the N protein of SARS-CoV- $2^{10}$. Furthermore, anti-SARS-CoV- 2 antibodies ${ }^{4}$ and CRP may also contribute to the activation of the classical and alternative pathways of complement during COVID-19. Thus, factors that trigger the activation of the complement pathways are upregulated in COVID-19 and may sustain the high levels of C5a that are detected in patients with severe COVID-19.

We found that COVID-19 was associated with peripheral blood neutrophilia (Fig. 2a), as reported in other cohorts ${ }^{11}$. No major changes were observed in the total peripheral blood monocyte population (Fig. 2b), but the proportion of conventional $\mathrm{CD} 14^{+} \mathrm{CD} 16^{-}$monocytes was increased, whereas the proportion of inflammatory $\mathrm{CD} 14^{\text {low }} \mathrm{CD} 16^{+}$ monocytes was decreased in peripheral blood (Fig. 2c), consistent with the possibility that inflammatory monocytes leave the bloodstream and home to tissues. This hypothesis was supported by transcriptomic analyses of patients with COVID-19 who were symptomatic, which revealed not only an increase in the transcript levels of genes including IRAK3, MS4A6A, CD33, CD3OOC, VCAN, CD1D, CCR1, OAS1, CD163 and C3AR1 in peripheral blood mononuclear cells, but also an upregulation of macrophage and monocyte transcriptomic signatures in inflamed lungs (Extended Data Fig. 1c).

Both circulating neutrophils and monocytes displayed strong C5aR1 expression in healthy individuals that was also observed in the various groups of patients with COVID-19 (Fig. 2d). Longitudinal immune-monitoring follow-up of patients with pneumonia and ARDS showed that the levels of $\mathrm{C} 5 \mathrm{aR} 1$ molecules remained stable on circulating neutrophils and monocytes, or even increased during the course of the disease (Extended Data Fig. 2a). Consistent with the inflammatory function of $\mathrm{C}_{5} \mathrm{a}^{12}$, and the expression of $\mathrm{C} 5 \mathrm{aR} 1$ on monocytes, $\mathrm{C} 5 \mathrm{a}$ increased the production of the inflammatory cytokines IL-6, TNF and CCL2 that is induced by lipopolysaccharide (LPS) on purified blood monocytes isolated from patients with COVID-19 (Fig. 2e). C5a also increased the production of cytokines by monocytes from patients with COVID-19 following stimulation with R848, which activates the TLR7 and/or TLR8 MyD88-dependent signalling pathway, mimicking the single-stranded RNA of SARS-CoV-2 (Fig. 2e).

Given that severe COVID-19 is associated with lung disease, we then focused on this organ. C5a was detected in the bronchoalveolar lavage fluid (BALF) of patients with COVID-19 who had ARDS (Fig. 3a). Inflammatory cytokines, such as CXCL8, CXCL9, CCL2 and-to a lesser extentCCL4, IL-6, TNF and IL-1 $\beta$, were also detected in these samples (Fig. 3a). Large numbers of neutrophils and monocytes were found in the BALF of patients with COVID-19 who had ARDS and these cells expressed C5aR1 (Fig. 3b). In addition, the analysis of single-cell RNA-sequencing data from healthy control individuals and patients with COVID-19 who had ARDS $^{13}$ revealed major changes in the myeloid cell population that infiltrated the lungs during the course of the SARS-CoV-2 infection. Indeed, the major myeloid cell subset (subset A) in healthy control individuals and the major myeloid cell subset (subset B) in patients with COVID-19 who had ARDS were clearly different (Fig. $3 \mathrm{c}$ and Extended Data Fig. 3a, b). The cells of subset B were characterized by higher transcript levels of inflammatory cytokine genes, such as CXCL8,CCL2, CCL4,CXCL9, TNF and IL-6 (Fig. 3d). Both myeloid cell subsets expressed C5AR1, although a slight upregulation was observed in subset $\mathrm{B}$ (Fig. $3 \mathrm{~d}$ ). A multiplex immunohistochemistry analysis of lungs from patients 


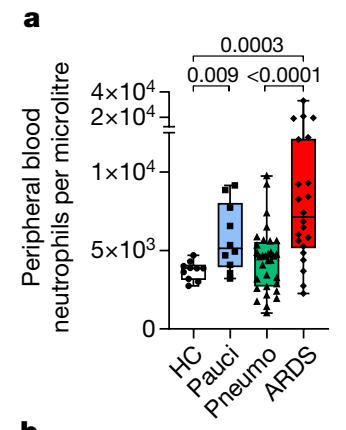

b
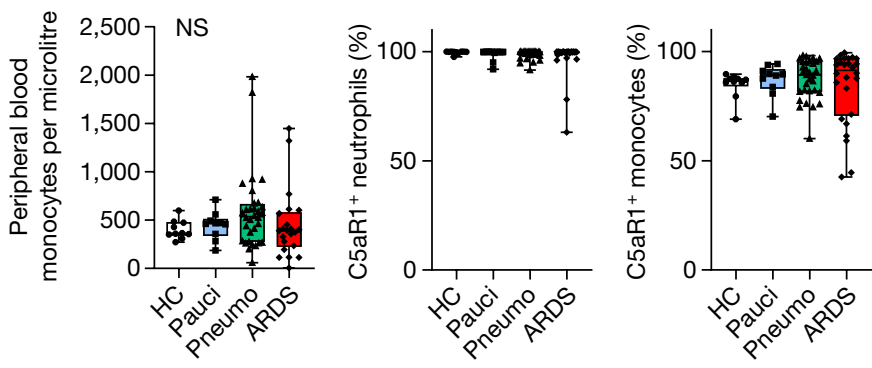

e
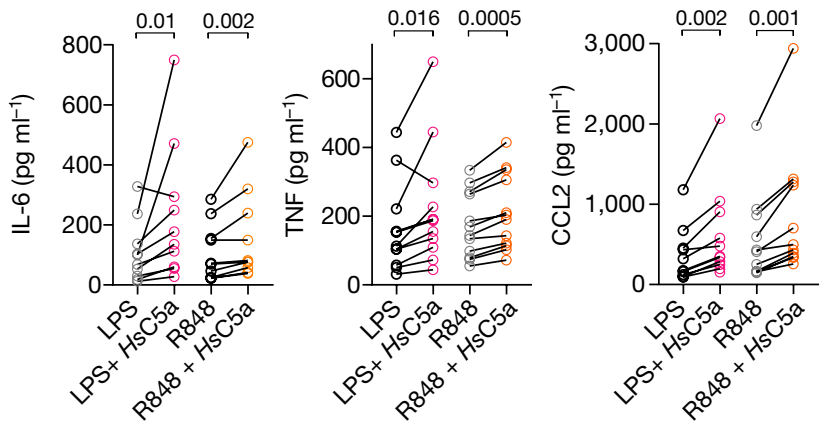

Fig. 2 |C5aR1 is highly expressed on myeloid cells and promotes inflammation. a, Absolute numbers of circulating neutrophils per microlitre of peripheral blood from healthy control individuals and patients with COVID-19 at $t_{0} \cdot \mathbf{b}$, Absolute numbers of circulating total monocytes per microlitre of peripheral blood from healthy control individuals and patients with COVID-19 at $t_{0}$.c. Percentages of $\mathrm{CD} 14^{+} \mathrm{CD} 16^{-}$conventional monocytes and $\mathrm{CD} 14^{\text {low }} \mathrm{CD} 16^{+}$inflammatory monocytes in the peripheral blood of healthy control individuals and patients with COVID-19 at $t_{0}$. d, Percentage of C5aR $1^{+}$ neutrophils and monocytes in peripheral blood from healthy control individuals and patients with COVID-19 at $t_{0} \cdot \mathbf{a}-\mathbf{d}$, Healthy control individuals, $n=10$; patients who were paucisymptomatic, $n=10$; patients with pneumonia, $n=31$; patients with ARDS, $n=26$. Each symbol represents a single donor. e, IL-6, TNF and CCL2 production by monocytes purified from peripheral blood mononuclear cells from patients with COVID-19. Peripheral blood mononuclear cells were activated overnight with LPS $\left(0.5 \mathrm{ng} \mathrm{ml}^{-1}\right)$ or R848 (50 $\left.\mathrm{ng} \mathrm{ml}^{-1}\right)$ and recombinant human $\mathrm{C} 5 \mathrm{a}(\mathrm{HsC} 5 \mathrm{a})\left(1 \mu \mathrm{g} \mathrm{ml}^{-1}\right)$, as indicated. Each dot represents the mean value obtained from duplicate or triplicate analyses for a single patient ( $n=12$ patients). Box plots show the median and the 25 th to 75 th percentiles, and the whiskers denote the maximum and minimum values. The $P$ values were obtained using two-tailed Wilcoxon rank-sum tests $(\mathbf{a}-\mathbf{d})$ and two-tailed Wilcoxon signed-rank tests (e). NS, no significant differences.

with ARDS with COVID-19 who were deceased confirmed pulmonary infiltration of $\mathrm{CD} 68^{+} \mathrm{CD} 163^{+}$macrophages, a substantial proportion of which expressed C5aR1 (Extended Data Fig. 3c (right)), relative to lung tissue from a control individual without COVID-19 (Extended Data Fig. 3c (left)). It is becoming increasingly clear that severe COVID-19 is associated with the spread of the virus through the epithelial barrier and with endothelialitis ${ }^{14-16}$. We observed obliterating endarteritis that was associated with an accumulation of $\mathrm{C} 5 \mathrm{aR} 1^{+}$macrophages around the arteries and in the thrombus (Extended Data Fig. 3d). Together with

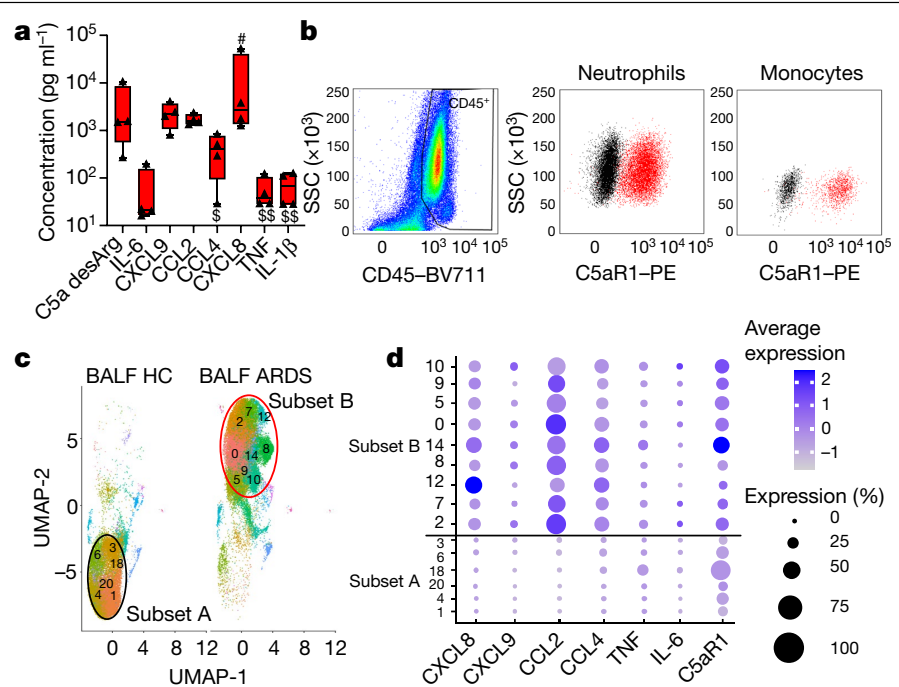

Fig. $3 \mid \mathrm{C5aR1}^{+}$cells and $\mathrm{C5a}$ are detected in lung samples from patients with COVID-19. a, Concentration of C5a desArg and IL-6, CXCL9, CCL2, CCL4, CXCL8, TNF and IL-1 $\beta$ in the BALF of patients with ARDS $(n=4)$. "Values were above the

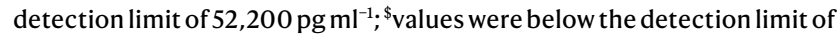
$29 \mathrm{pg} \mathrm{ml}^{-1}$. Box plots show the median and the 25 th to 75 th percentiles, and the whiskers denote the maximum and minimum values. b, Left, CD45 immune cell infiltration in BALF from patients with ARDS using flow cytometry. Right, C5aR1 expression (red) compared with negative control (fluorescence without antibody; black) staining on $\mathrm{CD} 45^{+} \mathrm{CD} 14^{-} \mathrm{CD} 15^{+} \mathrm{CD} 16^{+}$neutrophils and

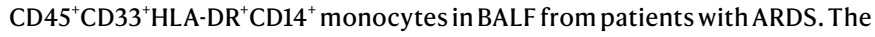
plots shown are representative of analyses performed on samples from three patients with ARDS.c, Uniform manifold approximation and projection (UMAP) analysis of myeloid cell clusters by patient group. Healthy control individuals, $n=3$; patients with severe COVID-19, $n=6$. d, Dot plot of pro-inflammatory cytokine expression in each of the subsets from $\mathbf{c}$.

the high levels of C5a in patients with COVID-19 who were symptomatic, these data support the hypothesis that C5a production leads to the chemo-attraction and activation of myeloid cells in the lungs, and contributes to the overt release of inflammatory cytokines. As C5a can also promote the secretion of CCL2, a strong chemoattractant for monocytes, C5a could also indirectly promote the recruitment of inflammatory cells indirectly, through the induction of other chemokines. It is also possible that the vasculitis associated with severe COVID-19 is linked to the production of $\mathrm{C} 5 \mathrm{a}$, as other types of vasculitis, such as anti-neutrophil cytoplasmic antibody (ANCA)-associated vasculitis, are mediated by $\mathrm{C} 5 \mathrm{a}^{17}$.

On the basis of this hypothesis, we reasoned that blockade of the C5a-C5aR1 axis could be used as a potential therapeutic strategy for the treatment of severe COVID-19. Several molecules could be repurposed to this end, including anti-C5 monoclonal antibodies, anti-C5a monoclonal antibodies and C5aR1 antagonists. We focused on avdoralimab, a fully human Fc-silent monoclonal antibody against C5aR1 that prevents its binding to $\mathrm{C} 5 \mathrm{a}$. In vitro, C5aR1 blockade with avdoralimab inhibited C5a-induced activation of neutrophils, as shown by evaluation of the induction of CD11b expression at the cell surface (Fig. 4a). The C5a-mediated upregulation of CD11b was also inhibited by other C5aR1 antagonists or anti-C5a monoclonal antibodies (Extended Data Fig. 4a). Avdoralimab blocked the activation of neutrophils induced by very high concentrations of $\mathrm{C} 5 \mathrm{a}$ (Fig. $4 \mathrm{~b}$ ). With regard to the infiltration of $\mathrm{C}^{\mathrm{aR}} \mathrm{I}^{+}$myeloid cells in the inflamed lungs of patients with severe COVID-19, avdoralimab also inhibited the C5a-induced migration of neutrophils in vitro (Fig. 4c). We next investigated whether avdoralimab could block the development of ALI in a mouse model. As avdoralimab targets human C5aR1, we used mice with a knock-in gene of human C5aR1 (HsC5AR1 knock-in mice) ${ }^{18}$ that showed HsC5aR1 expression 


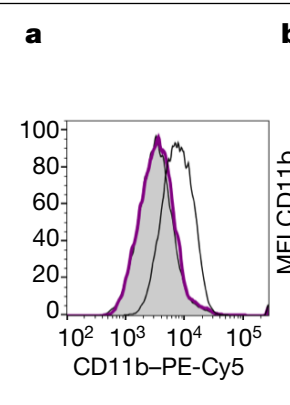

\section{b}
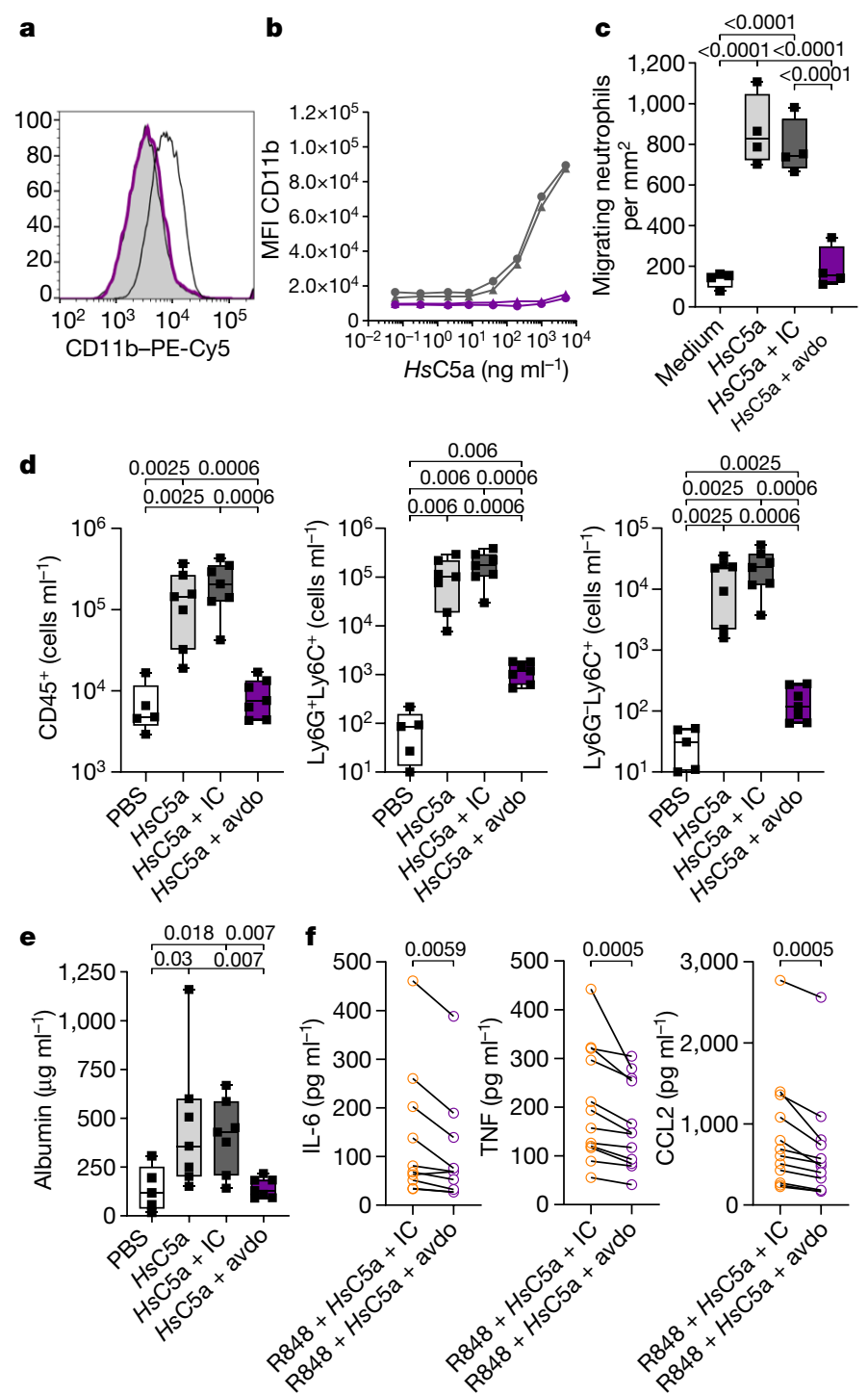

Fig. 4 | Targeting C5aR1 blocks the C5a-mediated activation and migration of myeloid cells. a, Representative flow cytometry histogram of the C5a-mediated upregulation of CD11b on whole-blood neutrophils, inhibited by avdoralimab. Grey, non-activated neutrophils; black, recombinant human C5a ( $H s \mathrm{C} 5 \mathrm{a}$ )-activated neutrophils; purple, $\mathrm{HsC}_{\mathrm{C}} \mathrm{a}$-activated neutrophils treated with avdoralimab $\left(30 \mu \mathrm{g} \mathrm{ml}^{-1}\right)$. b, CD11b induction through a dose-dependent response to $\mathrm{Hs}$ C5a on untreated (grey) or $10 \mu \mathrm{g} \mathrm{ml}^{-1}$ avdoralimab-treated whole-blood neutrophils (purple). MFI, median fluorescence intensity. Data were obtained from two independent donors. c, Migrating neutrophils attracted by $H s \mathrm{C} 5$ a. Neutrophils were treated with $10 \mu \mathrm{g} \mathrm{ml}^{-1}$ isotype control (IC) or avdoralimab (avdo) before the induction of migration by $\mathrm{HsC5a}$. Data for neutrophils purified from four healthy control individuals are shown. d, e, BALF was collected from HsC5AR1 knock-in mice $18 \mathrm{~h}$ after intranasal instillation of $H s C 5$ a. Mice were pretreated with avdoralimab or isotype control, as indicated. Each symbol represents data from a single mouse; $n=5$ mice (PBS) and $n=7$ mice (all other groups). Data are representative of two experiments. d, BALF cell analysis by flow cytometry of CD $45^{+}$immune cells, Ly $6 \mathrm{C}^{+} \mathrm{Ly} 6 \mathrm{G}^{+}$neutrophils and $\mathrm{Ly}_{6 \mathrm{C}^{+}} \mathrm{Ly} 6 \mathrm{G}^{-}$monocytes. e, Albumin concentration in BALF.f, Production of IL-6, TNF and CCL2 by monocytes purified from the peripheral blood mononuclear cells of patients with COVID-19 activated by overnight incubation with R848 $\left(50 \mathrm{ng} \mathrm{ml}^{-1}\right)$ and $\mathrm{HsC} 5 \mathrm{a}\left(1 \mu \mathrm{g} \mathrm{ml}^{-1}\right)$. Before activation, monocytes were incubated with $20 \mathrm{\mu g} \mathrm{ml}^{-1}$ avdoralimab or isotype control. Each dot represents the mean value obtained from duplicate or triplicate analyses of a single donor; $n=10$ (IL-6) and $n=12$ (TNF and CCL2). Box plots show the median and the 25 th to 75 th percentiles, and the whiskers denote the maximum and minimum values. The $P$ values were computed using a paired one-way analysis of variance (ANOVA) (c), two-tailed Wilcoxon rank-sum tests (d,e) and two-tailed Wilcoxon signed-rank tests (f). exclusively on CD11b ${ }^{+}$myeloid cells (Extended Data Fig. 4b). HsC5AR1 knock-in mice that received an intranasal instillation of recombinant human C5a developed ALI and injury $18 \mathrm{~h}$ after injection, as shown by the infiltration of $\mathrm{CD} 45^{+}$immune cells including $\mathrm{Ly}_{6 \mathrm{G}}{ }^{+} \mathrm{Ly} 6 \mathrm{C}^{+}$neutrophils and $\mathrm{Ly}_{6 \mathrm{G}} \mathrm{Ly}^{\mathrm{L}} 6 \mathrm{C}^{+}$monocytes in the lung (Fig. $4 \mathrm{~d}$ ) and the release of albumin in BALF (Fig. 4e), a marker of alveolar-capillary permeability and lung injury. ALI was confirmed by histopathological analysis of lung sections that revealed massive inflammatory cell infiltration, alveolar haemorrhage and thickening of alveolar walls in lungs of C5a-treated mice (Extended Data Fig. 4c), as previously described ${ }^{9}$. Avdoralimab blocked the infiltration of both cell types (Fig. 4d), prevented albumin release in BALF (Fig. 4e) and limited C5a-induced ALI histopathological features (Extended Data Fig. 4c). Finally, avdoralimab also inhibited the increase in IL-6, TNF and CCL2 secretion induced in vitro by C5a in monocytes purified from patients with COVID-19 and activated with a single-strand RNA-virus-like stimulus (Fig. 4f). Therefore, avdoralimab seems to be suitable for blocking the C5a-C5aR1 axis, which is active during COVID-19. The high levels of C5a observed in patients with COVID-19 do not appear to be a passenger phenomenon, as preliminary efficacy data reported that two patients with COVID-19 recovered from ARDS after treatment with an anti-C5a blocking monoclonal antibody $(\text { IFX-1 })^{10}$. Furthermore, four patients with severe COVID-19 treated with an anti-C5 monoclonal antibody (eculizumab) exhibited a drop in circulating inflammatory markers ${ }^{19}$. There are several advantages to blocking $\mathrm{C} 5 \mathrm{aR} 1$ rather than other components of the complement cascade. First, blocking C5a or C5aR1 leaves C5b intact and preserves the MAC, which has a key role in controlling several infections. A loss of the MAC would raise safety concerns for the treatment of COVID-19, as patients who are symptomatic often develop comorbid conditions, such as bacterial infections, for which the MAC is required. Second, C5aR1 blockade has the advantage over C5a blockade of having no effect on the second C5a receptor, C5L2. The function of C5L2 remains unclear, but it has been suggested that it can act as a decoy receptor, with anti-inflammatory roles ${ }^{20}$.

Our data, which highlight the role of the C5a-C5aR1 axis in the pathogenesis of severe COVID-19, are consistent with several previous observations. Indeed, high C5a levels have also been described in various preclinical models of acute lung disease due to pathogenic viruses, such as SARS-CoV, H1N1, H5N1 and H7N9 ${ }^{1}$. High levels of C5a have also been found in the upper respiratory tract and in serum samples from patients infected with $\mathrm{H} 1 \mathrm{~N} 1^{21}$. Furthermore, BALF from patients with ARDS was found to display robust C5a-dependent chemotactic activity ${ }^{22}$. In a mouse model of Middle East respiratory syndrome coronavirus (MERS-CoV) infection, C5a concentrations were high in serum samples and lung tissues ${ }^{23}$, and anti-C5aR1 antibody treatment decreased viral replication in lung tissue ${ }^{23}$. In a green monkey model of $\mathrm{H} 7 \mathrm{~N} 9$ infection, treatment with an anti-C5a antibody significantly decreased the levels of IL-6, interferon- $\gamma$, TNF and IL-1 $\beta$ and neutrophil infiltration in the lungs ${ }^{24}$. Overall, C5a inhibition markedly decreased the ALI and systemic inflammation induced by viral infection ${ }^{25}$. Further evidence for the involvement of the complement system in the pathogenesis of severe COVID-19 is provided by the existence of genetic variants of the complement cascade, which are associated with clinical outcome of SARS-CoV-2 infection, such as $C D 55^{26}$.

In addition, the complement system links innate immunity to coagulation $^{27,28}$, and its overactivation could promote thrombotic events in patients with severe COVID-1929. Complement blockade may, therefore, prevent thrombosis in affected individuals.

The data presented here support a role for the $\mathrm{C} 5 \mathrm{a}-\mathrm{C} 5 \mathrm{aR} 1 \mathrm{axis}$ in the inflammatory mechanisms that underlie the development of ARDS in patients at early or late stages of infection with SARS-COV-2, which is consistent with other reports ${ }^{10,30}$. As described above, in addition to pneumonia and ARDS, there are data that suggest a role of C5a in other COVID-19-related symptoms, including heart, kidney or endothelial 
cell dysfunction ${ }^{14}$, providing support for the testing of the blockade of the C5a-C5aR1 axis in patients with COVID-19. We suggest that such a blockade may prevent the transition from a localized epithelial disease (non-severe COVID-19) to a diffuse endothelial disease (severe COVID-19) (Extended Data Fig. 4d).

\section{Online content}

Any methods, additional references, Nature Research reporting summaries, source data, extended data, supplementary information, acknowledgements, peer review information; details of author contributions and competing interests; and statements of data and code availability are available at https://doi.org/10.1038/s41586-020-2600-6.

1. Wang, R., Xiao, H., Guo, R., Li, Y. \& Shen, B. The role of C5a in acute lung injury induced by highly pathogenic viral infections. Emerg. Microbes Infect. 4, 1-7 (2015).

2. Cao, X. COVID-19: immunopathology and its implications for therapy. Nat. Rev. Immunol. 20, 269-270 (2020).

3. Mehta, P. et al. COVID-19: consider cytokine storm syndromes and immunosuppression. Lancet 395, 1033-1034 (2020).

4. Long, Q. X. et al. Antibody responses to SARS-CoV-2 in patients with COVID-19. Nat. Med 26, 845-848 (2020)

5. Guo, R. F. \& Ward, P. A. Role of C5a in inflammatory responses. Annu. Rev. Immunol. 23, 821-852 (2005).

6. Bosmann, M. \& Ward, P. A. Role of $\mathrm{C} 3, \mathrm{C} 5$ and anaphylatoxin receptors in acute lung injury and in sepsis. Adv. Exp. Med. Biol. 946, 147-159 (2012).

7. Ricklin, D., Hajishengallis, G., Yang, K. \& Lambris, J. D. Complement: a key system for immune surveillance and homeostasis. Nat. Immunol. 11, 785-797 (2010).

8. Guo, R. F., Riedemann, N. C. \& Ward, P. A. Role of C5a-C5aR interaction in sepsis. Shock 21, 1-7 (2004).

9. Russkamp, N. F. et al. Experimental design of complement component 5a-induced acute lung injury (C5a-ALI): a role of CC-chemokine receptor type 5 during immune activation by anaphylatoxin. FASEB J. 29, 3762-3772 (2015).

10. Gao, T. et al. Highly pathogenic coronavirus $N$ protein aggravates lung injury by MASP2-mediated complement over-activation. Preprint at medRxiv https://doi.org/10.1101/ 2020.03.29.20041962 (2020)

11. Liu, J. et al. Neutrophil-to-lymphocyte ratio predicts critical illness patients with 2019 coronavirus disease in the early stage. J. Transl. Med. 18, 206 (2020).

12. Riedemann, N. C. et al. Increased C5a receptor expression in sepsis. J. Clin. Invest. 110 101-108 (2002).

13. Liao, M. et al. Single-cell landscape of bronchoalveolar immune cells in patients with COVID-19. Nat. Med. 26, 842-844 (2020).

14. Varga, Z. et al. Endothelial cell infection and endotheliitis in COVID-19. Lancet 395, 1417-1418 (2020).

15. Teuwen, L.-A., Geldhof, V., Pasut, A. \& Carmeliet, P. COVID-19: the vasculature unleashed. Nat. Rev. Immunol. 20, 389-391 (2020).

16. Copin, M. C. et al. Time to consider histologic pattern of lung injury to treat critically ill patients with COVID-19 infection. Intensive Care Med. 46, 1124-11126 (2020).

17. Jayne, D. R. W. et al. Randomized trial of C5a receptor inhibitor avacopan in ANCA-associated vasculitis. J. Am. Soc. Nephrol. 28, 2756-2767 (2017).

18. Lee, H. et al. Human C5aR knock-in mice facilitate the production and assessment of anti-inflammatory monoclonal antibodies. Nat. Biotechnol. 24, 1279-1284 (2006).
19. Diurno, F. et al. Eculizumab treatment in patients with COVID-19: preliminary results from real life ASL Napoli 2 Nord experience. Eur. Rev. Med. Pharmacol. Sci. 24, 4040-4047 (2020).

20. Gerard, N. P. et al. An anti-inflammatory function for the complement anaphylatoxin C5a-binding protein, C5L2. J. Biol. Chem. 280, 39677-39680 (2005).

21. Bjornson, A. B., Mellencamp, M. A. \& Schiff, G. M. Complement is activated in the upper respiratory tract during influenza virus infection. Am. Rev. Respir. Dis. 143, 1062-1066 (1991).

22. Trujillo, G. et al. Cofactor regulation of C5a chemotactic activity in physiological fluids. Requirement for the vitamin $\mathrm{D}$ binding protein, thrombospondin-1 and its receptors. Mol. Immunol. 49, 495-503 (2011).

23. Jiang, Y. et al. Blockade of the C5a-C5aR axis alleviates lung damage in hDPP4-transgenic mice infected with MERS-CoV. Emerg. Microbes Infect. 7, 1-12 (2018).

24. Sun, S. et al. Treatment with anti-C5a antibody improves the outcome of H7N9 virus infection in African green monkeys. Clin. Infect. Dis. 60, 586-595 (2015).

25. Sun, S. et al. Inhibition of complement activation alleviates acute lung injury induced by highly pathogenic avian influenza H5N1 virus infection. Am. J. Respir. Cell Mol. Biol. 49, 221-230 (2013).

26. Ramlall, V. et al. Immune complement and coagulation dysfunction in adverse outcomes of SARS-CoV-2 infection. Nat. Med. https://doi.org/10.1038/s41591-020-1021-2 (2020).

27. Foley, J. H. Examining coagulation-complement crosstalk: complement activation and thrombosis. Thromb. Res. 141, S50-S54 (2016).

28. Thomson, T. M., Toscano, E., Casis, E. \& Paciucci, R. C1 esterase inhibitor and the contact system in COVID-19. Br J. Haematol. 190, 520-524 (2020).

29. Magro, C. et al. Complement associated microvascular injury and thrombosis in the pathogenesis of severe COVID-19 infection: a report of five cases. Transl. Res. 220, 1-13 (2020).

30. Cugno, M. et al. Complement activation in patients with COVID-19: a novel therapeutic target. J. Allergy Clin. Immunol. 146, 215-217 (2020).

Publisher's note Springer Nature remains neutral with regard to jurisdictional claims in published maps and institutional affiliations.

(c) The Author(s), under exclusive licence to Springer Nature Limited 2020

Explore COVID-19 IPH group

Laura Assante Miranda ${ }^{3}$, William Baron ${ }^{3}$, Nourhène Belaid ${ }^{3}$, Clarisse Caillet ${ }^{3}$, Flavien Caraguel $^{3}$, Barbara Carrette ${ }^{3}$, Florent Carrette ${ }^{3}$, Fabien Chanuc ${ }^{3}$, Rachel Courtois ${ }^{3}$, Aurore Fenis $^{3}$, Marilyn Giordano ${ }^{3}$, Mathilde Girard-Madoux ${ }^{3}$, Marc Giraudon-Paoli ${ }^{3}$, Nicolas Gourdin $^{3}$, Gwendoline Grondin ${ }^{3}$, Franceline Guillot ${ }^{3}$, Guillaume Habif ${ }^{3}$, Solène Jaubert ${ }^{3}$, Julie Lopez ${ }^{3}$, Mélanie Le Van $^{3}$, Naouel Lovera ${ }^{3}$, Marine Mansuy ${ }^{3}$, Elodie Bonnet ${ }^{3}$, Audrey Sansaloni ${ }^{3}$, Annick Reboul ${ }^{3}$, Emmanuel Mitry ${ }^{3}$, Camille Nekkar-Constant ${ }^{3}$, Valentine Péri ${ }^{3}$, Paul Ricaut ${ }^{3}$, Léa Simon ${ }^{3}$, Jean-Baptiste Vallier ${ }^{3}$, Marie Vétizou ${ }^{3} \&$ Robert Zerbib ${ }^{3}$

Explore COVID-19 Marseille Immunopole group

Sophie Ugolini ${ }^{4}$, Marion Etiennot ${ }^{4}$, Justine Galluso ${ }^{4}$, Luc Lyonnet ${ }^{7}$, Jean-Marie Forel ${ }^{7}$, Laurent Papazian ${ }^{7}$, Lionel Velly ${ }^{7}$, Baptiste André ${ }^{7}$, Antoine Briantais ${ }^{7}$, Benoit Faucher ${ }^{7}$, Estelle Jean ${ }^{7}$, Julie Seguier ${ }^{7}$, Veronique Veit ${ }^{7}$, Jean-Robert Harlé ${ }^{7}$, Boris Pastorino ${ }^{7}$, Clémence Delteil ${ }^{7}$, Laurent Daniel ${ }^{7}$, Jean-Paul Boudsocq ${ }^{6}$, Axelle Clerc $^{6}$, Emmanuel Delmond ${ }^{6}$, Pierre-Olivier Vidal $^{6}$, Hélène Savini ${ }^{6}$ \& Bruno Coutard"

${ }^{11}$ Unité des Virus Émergents (UVE), Aix-Marseille Université, IRD 190, Inserm 1207, IHU Méditerranée Infection, Marseilles, France. 


\section{Methods}

\section{Data reporting}

No statistical methods were used to predetermine sample size. The experiments were not randomized and the investigators were not blinded to allocation during experiments and outcome assessment.

\section{Study participants and clinical considerations}

Over a period of one month (27 March 2020-24 April 2020), 82 participants were recruited from three hospitals (Timone and Nord University Hospitals, and Laveran Military Hospital, Marseille). Of the 82 participants, 28 were on mechanical ventilation for COVID-19-related-ARDS $(P / F$ ratio $<300)$ (ARDS group), 34 patients required oxygen support at a rate of less than $51 \mathrm{~min}^{-1}$ for COVID-19-related pneumonia (pneumonia group) and 10 patients had a paucisymptomatic form of COVID-19 that was compatible with outpatient care (paucisymptomatic group). COVID-19 was diagnosed on the basis of positive SARS-CoV-2 real-time reverse-transcription PCR (RT-PCR) on nasopharyngeal samples and/ or typical CT-scan findings ${ }^{31}$. We also included 10 healthy volunteers (control group), with no fever or symptoms on the days before sampling and negative for SARS-CoV-2RT-PCR. The characteristics of the patients are described in Supplementary Table 1. Biological samples were first collected within 3 days of diagnosis and the start of care $\left(t_{0}\right.$, $<72 \mathrm{~h}$, early time point). When possible, the next two time points for sample collection were located between days 5 and $10\left(t_{1}\right.$, intermediate time point) and after day $10\left(t_{2}\right.$, late time point). Flow cytometry analyses were performed on fresh blood samples (EDTA tubes) and BALFs, immediately after collection. Clinical progression was evaluated between the early and intermediate time points and between the intermediate and late time points. A favourable outcome was defined as weaning from mechanical ventilation (ARDS group) or oxygen support (pneumonia group). Death or multiple organ failure (ARDS group) and admission to the intensive care unit (pneumonia group) were considered unfavourable outcomes. In other cases, patients were considered to be stable.

\section{Ethics approval statement}

All of the patients (and/or initially their families) provided written informed consent before sampling and for the use of their clinical and biological data. The study protocol was approved on 27 March 2020 by the Committee for the Protection of Persons Ile-de-France III - France (2020-A00757-32). The pathological examination used in this study was performed secondary to a medical autopsy after COVID-19-related death, with agreement from the family and notification of the representative of the Commission on Data Processing and Freedom (MR003 research).

\section{Animals}

Female C57BL/6J mice were purchased at Janvier Labs and used between 8 and 12 weeks of age. HsC5AR1 knock-in mice were bred at Charles River Laboratories under specific-pathogen-free conditions. Female mice were used at 8-12 weeks of age and were allowed to acclimatize to the housing facility for at least one week. All animal experiments were performed in accordance with the rules of the Innate Pharma ethics committee and were approved by the Ministère de l'Enseignement Supérieur, de la Recherche et de l'Innovation - France (APAFIS\#25418-2020051512242806 v2).

\section{Reagent list}

DPBS (1×) (14190-094, Gibco); RPMI medium 1640 (1×) (31870025, Gibco); sodium pyruvate $100 \mathrm{mM}(100 \times)$ (11360-039, Gibco); L-glutamine $200 \mathrm{mM}(100 \times)$ (25030-024, Gibco); minimum essential medium non-essential amino acids solution (11140-035, Gibco); trypan blue stain (0.4\%) (15250-061, Gibco); Ficoll-Paque PLUS (17-1440-03, GE Healthcare); fetal calf serum (FCS; F7524, Sigma); dimethyl sulfoxide (DMSO; D2650-100ML, Sigma); CD14 microbeads human (130-050-201, Miltenyi Biotec); EasySep direct human neutrophil isolation kit (19666, StemCell Technologies); bovine serum albumin (BSA; A9418-100G, Sigma); UltraPure 0.5 M EDTA, pH 8.0 (15575-038, Invitrogen); LPS EK ultrapure (tlrl-peklps, Invivogen); R848 (tlrl-r848, Invivogen); C5a (IPH 1D9 batch 1A, Innate Pharma); C5a (2037-C5-025, R\&D Systems); avdoralimab (Innate Pharma), isotype control (FC-silent hIgG1) (Innate Pharma); CD33-PECF594 clone WM53 (562492, BD Biosciences); CD19-PECy7 clone SJ25C1(557835, BD Biosciences); CD3-BUV496 clone UCHT1(564809/612940, BD Biosciences); CD15-BV510 clone W6D3 (563141, BD Biosciences); CD45BV711 clone HI30 (564357, BD Biosciences); CD16-BUV395 clone 3G8 (563785, BD Biosciences); CD14-BUV737 clone M5E2 (564444/612763, BD Biosciences); HLA-DR-AF700 clone L243 (307626, BioLegend); LIVE/ DEAD Near-IR (L34976, ThermoFisher); mouse serum (015-000-120, Jackson ImmunoResearch); CD88 C5aR PE clone S5/1 (344304, BioLegend); anti-CD16-FITC (556616, BD Biosciences); anti-CD11b-PE-Cy5 (555389, BD Biosciences); U-PLEX kit (NO5235A-1, MSD); OptEIA HsC5a ELISA (557965, BD Biosciences); mouse albumin ELISA kit (E99-134, Bethyl Laboratories); Ficoll (11778538, Invitrogen); dextran (31382, Sigma); calcein AM (C3100MP, Invitrogen); fibrinogen (F3879, Sigma); Transwell Fluoroblok $3 \mu \mathrm{m}$ insert (351151, Corning); EDTA (15575-038, Invitrogen); sodium azide (71290-100g, Sigma); Optilyse C solution (A11895, Beckman Coulter); CytoFix (554655, BD Bioscience); avacopan (HY-17627, Clinisciences); anti-C5a (Innate Pharma); anti-mouse Ly6CBV510 clone HK1.4 (128033, BioLegend); anti-mouse Ly6G-BV786 clone 1A8 (740953, BD Biosciences); anti-mouse CD45-BUV395 clone 30F11 (564279, BD Biosciences), anti-mouse CD11b-BUV737 clone M1/70 (564443/612800, BD Biosciences); anti-mouse C5aR1-APC clone 20/70 (130-106-124, Miltenyi Biotec); anti-human CD88 clone S5/1 (HM2094100UG, Hycult Biotech); anti-human CD68 clone KP1 (M0814, Agilent); anti-human CD163 clone EDHu-1 (MCA1853, BioRad).

\section{SARS-CoV-2 detection by PCR}

SARS-CoV-2 RNA was detected by RT-PCR, as previously described ${ }^{32}$.

\section{Seroneutralization assay}

Experiments were performed in BSL3 facilities with a clinical isolate of SARS-CoV-2. Virus-neutralization tests were performed as previously described ${ }^{33}$. In brief, virus-neutralization tests were performed in a 96-well plate, with Vero E6 cells and a SARS-CoV-2 strain (Ref-SKU:026V-03883 isolated at Charité University, Berlin, Germany; EVA-GLOBAL H2020 project; grant agreement 871029). Twofold serial dilutions of serum samples (final serum dilutions of 1:20 to 1:160) were mixed with a median tissue culture infective dose of 100 of SARS-CoV2 and dispensed on the confluent cell monolayer. The plates were incubated for 4 days and examined for the presence (no neutralization) or absence (neutralization) of cytopathogenic effects under an inverted microscope.

\section{Preparation of peripheral blood mononuclear cells and plasma} Whole blood collected in EDTA tubes was pooled and diluted 1:2 in PBS. Peripheral blood mononuclear cells (PBMCs) were isolated by centrifugation on a Ficoll gradient, and $10^{7} \mathrm{PBMCs}$ per vial were frozen in freezing medium (90\% FCS and $10 \%$ DMSO). Plasma was collected from the upper phase of the Ficoll gradient, aliquoted and used for the quantification of soluble factors.

\section{Assessment of soluble factors}

Human IL-6, CXCL9, CCL2, CCL4, CXCL8, TNF and IL-1 $\beta$ levels were analysed with the U-PLEX kit supplied by MSD (U-PLEX 10-Assay, 96-Well SECTOR plate, N05235A-1), according to the manufacturer's instructions. The U-PLEX plate was loaded into an MSD instrument to measure the intensity of emitted light, which is proportional to the amount of analyte present in the sample. Circulating C5a desArg levels were 
analysed using a BD OptEIA $H s C 5$ a ELISA. Mouse albumin in BALF was analysed by ELISA (Bethyl). HRP-conjugated secondary antibodies were detected by incubation with a peroxidase substrate solution (TMB) and the reaction was stopped by acidification. Plates were read at $450 \mathrm{~nm}$.

\section{Flow cytometry}

Blood collected into EDTA tubes was washed in PBS before staining with LIVE/DEAD (Thermo Fisher) according to the manufacturer's instructions. Cells were incubated with mouse serum to saturate the Fc receptors, and were then incubated with the appropriate antibody cocktail. Red blood cells were lysed in Optilyse C solution (Beckman Coulter), according to the manufacturer's instructions. Cells were fixed in CytoFix solution (BD Bioscience, 554655), according to the manufacturer's instructions. Data were acquired in an LSRFortessaX20 flow cytometer. The FCS3.0 files obtained were exported from BDFACSDiva software and imported into FlowJo v.10.5.2 (BD Biosciences). Automated compensation was calculated with FACSDiva software and single-antibody-stained compensation beads. This compensation matrix was analysed in detail in FlowJo, by investigating the $\mathrm{N}-b y-\mathrm{N}$ view feature and the pairwise expression of all proteins stained in this study. Fluorescence minus one experiments were run before this study, to facilitate optimization of the compensation matrix. We then adjusted the compensation matrix where necessary due to over- or under-compensation by the automatic algorithm.

\section{Immune cell counts}

Absolute counts per microlitre of blood were determined with BD TBNK Trucount Tubes. Absolute counts for a particular cell population $(A)$ were obtained by dividing the number of positive cell events $(X)$ by the number of bead events $(Y)$, and then multiplying by the BD Trucount bead concentration $(N / V$, where $N$ is the number of beads per test and $V$ is the test volume). $A=X / Y \times N / V$. The number of positive counts for neutrophils and monocytes was established with the $\mathrm{CD} 45^{+} \mathrm{SSC}^{\text {high }}$ and $\mathrm{CD}^{+} 5^{+} \mathrm{SSC}^{\text {int }}$ gating strategies, respectively.

\section{C5a inhibitors}

Avdoralimab is a fully human mutated Fc-silent IgG1 monoclonal antibody against C5aR1 (US 2020/0017599A1). Anti-C5a monoclonal antibody is a chimeric mutated Fc silent IgG1 isotype cloned from the sequences of mouse anti-HsC5a INab308 (WO2015/140304A1), and has the same variable sequences as IFX-1. The C5aR1 antagonist (avacopan) was purchased from Clinisciences (HY-17627).

\section{Neutrophil migration}

Neutrophils were isolated from fresh blood by sedimentation in $6 \%$ dextran to separate plasma and leukocytes, followed by centrifugation on a Ficoll density gradient. The pellet, containing neutrophils, was recovered, and the red blood cells were lysed by incubation in $0.2 \% \mathrm{NaCl}$. Osmotic balance was restored by adding an equal volume of $1.6 \% \mathrm{NaCl}$. Isolated neutrophils were loaded with $10 \mu \mathrm{M}$ calcein AM. Cell density was adjusted before the addition of avdoralimab or its isotype control at a final concentration of $10 \mu \mathrm{g} \mathrm{ml}^{-1}$. Neutrophils were dispensed into the top chamber of a fibrinogen- and BSA-coated Transwell Fluoroblok $3 \mu \mathrm{m}$ insert. The lower chamber was filled with RPMI 1640 with or without $3 \mathrm{nM} \mathrm{HsC5a}$ (R\&D Systems,) and the same antibody was added to the top chamber (avdoralimab, isotype control or PBS). After $30 \mathrm{~min}$ of incubation at $+37 \pm 1{ }^{\circ} \mathrm{C}$ under an atmosphere containing $5 \pm 1 \% \mathrm{CO}_{2}$, images of the bottom side of the inserts were acquired on a Biotek Cytation 5 plate-reading microscope, and analysed with Halo software (Indica Labs), using the CytoNuclear FL module to count the cells that had crossed the membrane.

\section{Neutrophil activation}

Various concentrations of avdoralimab were added to the blood samples in culture-treated 96-well U-bottom plates, and incubated for $20 \mathrm{~min}$ at $37^{\circ} \mathrm{C}$ under an atmosphere containing $5 \% \mathrm{CO}_{2}$. We then added $18 \mathrm{nM} \mathrm{HsC5a}$ (R\&D Systems) to the samples. Plates were incubated for $20 \mathrm{~min}$ at $37^{\circ} \mathrm{C}$ under an atmosphere of $5 \% \mathrm{CO}_{2}$. Samples were then stained for flow cytometry analysis with anti-CD16-FITC and anti-CD11b-PE-Cy5 antibodies. Erythrocytes were lysed with Optilyse C solution (Beckman Coulter, A11895), according to the manufacturer's protocol, and resuspended in CytoFix (BD Bioscience 554655) for fixation. Cells were then analysed on a FACS Canto II flow cytometer (BD Biosciences) with FACS Diva software.

\section{Monocyte activation}

Monocytes were purified with the $\mathrm{CD} 14^{+}$selection kit (Miltenyi). We used 30,000 monocytes to seed 96-well U-bottom plates. Cells were activated by overnight incubation with $\mathrm{R} 848\left(50 \mathrm{ng} \mathrm{ml}^{-1}\right)$, LPS ( $\left.0.5 \mathrm{ng} \mathrm{ml}^{-1}\right)$ and $\mathrm{HsC} 5 \mathrm{a}\left(1 \mu \mathrm{g} \mathrm{mL}^{-1}\right.$; IPH). In some conditions, monocytes were incubated with avdoralimab $\left(20 \mu \mathrm{g} \mathrm{ml}^{-1}\right.$; IPH) or its isotype control $\left(20 \mu \mathrm{g} \mathrm{ml}^{-1}\right.$; IPH) for 30 min before stimulation. IL-6, TNF and CCL2 levels were quantified in the supernatant.

\section{Mouse model of lung inflammation}

Isoflurane-anaesthetized HsC5AR1 knock-in mice received 3.1 $\mu \mathrm{g}$ of recombinant $H s C 5 a(R \& D)$ in $40 \mu \mathrm{l}$ PBS, by intranasal instillation. After $18 \mathrm{~h}$, mice were killed with a lethal dose of ketamine/xylazine cocktail (ketamine $300 \mathrm{mg} \mathrm{kg}^{-1}$; xylazine $30 \mathrm{mg} \mathrm{kg}^{-1}$ ). The lungs were flushed with $2 \mathrm{ml} 2 \mathrm{mM}$ EDTA in PBS to obtain BALF. After centrifugation $\left(300 \mathrm{~g}, 5 \mathrm{~min}, 4^{\circ} \mathrm{C}\right.$ ), BALF cells were counted and stained for flow cytometry analysis with anti-CD45, anti-Ly6C, anti-Ly6G and anti-CD11b antibodies. For histology analysis, $18 \mathrm{~h}$ after intranasal instillation of $\mathrm{HsC}$ a, lungs were fixed in formalin, dissected, embedded in paraffin and sectioned to $5 \mu \mathrm{m}$. Sections were dewaxed and stained with haematoxylin and eosin (H\&E). Slides were scanned using a Nanozoomer S60 (Hamamatsu) and examined for evidence of lung damage.

\section{Multiplex immunohistochemistry staining protocol, image acquisition and data analysis}

Multiplexed immunohistochemistry (IHC) was performed with a Leica Bond Rx on 5- $\mu \mathrm{m}$-thick formalin-fixed paraffin-embedded lung tissue sections from individuals with and without COVID-19. Consecutive staining was performed by heat-induced antigen retrieval followed by incubation with primary antibody (anti-C5aR1 clone S5/1 at $1 \mu \mathrm{g} \mathrm{ml}^{-1}$ ). The signal was amplified and detected with Opal polymer horseradish peroxidase and Opal 520 (Akoya Biosciences). The sections were then subjected to heat-induced antibody stripping and incubated with the next antibody (anti-CD163 clone EDHu-1 at $1 \mu \mathrm{g} \mathrm{ml}^{-1}$, detected with Opal 620 , and, finally, anti-CD68 clone KP1 at $0.1 \mu \mathrm{g} \mathrm{ml}^{-1}$, detected with Opal 690) and spectral DAPI. All Opal reagents were used at a dilution of 1:150. Slides were mounted in ProLong Diamond anti-fade mounting medium (Thermo Fisher) and scanned with a Vectra Polaris (Akoya Biosciences). H\&E-stained slides were scanned with a Nanozoomer (Hamamatsu). After spectral deconvolution and whole-slide reconstruction of the multiplexed IHC stained sections, digital pathology methods were used to determine the density of positive cells. All analyses were performed with Halo (Indica Labs) and R.

\section{Transcriptomic analysis}

Transcriptomic analyses were performed on previously reported data $^{13,34}$. The RNA-sequencing (RNA-seq) data for two BALF samples from patients (each in duplicate), three PBMC samples from healthy control individuals and three PBMC samples from patients were downloaded from the National Genomics Data Center (https://bigd.big. ac.cn/; accession number PRJCA002326). The RNA-seq data for three BALF samples from healthy control individuals were downloaded from the SRA database (accession numbers, SRR10571724, SRR10571730 and SRR10571732). 
RNA-seq pipeline. The reads were mapped to the human genome (hg38) release 96 from Ensembl with STAR ${ }^{35}$. PCR replicates mapping to the human genome were removed with the Picard MarkDuplicates program (Broad Institute 2019, http://broadinstitute.github.io/picard/). Gene expression was calculated with featureCounts in the SubReads package (v.1.6.4 $)^{36}$. Transcripts per million values were calculated from the raw counts and $\log _{2}$-transformed. The depth of sequencing of the patient BALF samples was low ( $<1$ million).

Batch effect correction. We corrected for the batch effect between the datasets for BALF samples from healthy control individuals and those from patients and PBMC samples with Combat ${ }^{37}$, using the model: $\sim$ Batch + Status (Patient or Healthy) + Sample Type (PMBC or BALF). An analysis of differential expression between PBMC samples from healthy control individuals and patients was performed on raw counts with DESeq $2^{38}$. Significance was defined as an adjusted $P<0.05$. The metagene IRAK3 MS4A6A, CD33, CD3OOC, VCAN, CD1D, CCR1, OAS1, CD163, $C D 14, F C N 1, A I F 1$ and $P L A 2 G 7$ was used to calculate the macrophage and monocyte transcriptomic signature. The significance of the difference between healthy control individuals and patients was evaluated using a Wilcoxon rank-sum test. The single-cell RNA-seq data for 12 BALF samples from three healthy control individuals, three patients with mild COVID-19 and six patients with severe COVID-19 were downloaded from the Gene Expression Omnibus (GEO) database (accession number GSE145926). Quality controls were applied to each cell, for all samples, with the same criteria as for the initial analysis (gene number between 200 and 6,000 , UMI count $>1,000$ and mitochondrial gene percentage $<0.1$ ) with the Seurat package (v.3.1.0). After filtering, 63,740 cells were validated. As previously described ${ }^{13}$ the filtered gene-barcode matrix was first normalized with 'LogNormalize' methods in Seurat v.3, with default parameters. The top 2,000 variable genes were then identified by the 'vst' method with the Seurat FindVariableFeatures function. The variables 'nCount_RNA' and 'percent.mito' were regressed out in the scaling step and a principal component analysis was performed on the top 2,000 variable genes. For the reanalysis presented here, the batch effects across different donors were removed by Harmony ${ }^{39}$ and UMAP was performed on the top 50 dimensions for visualizing the cells. Graph-based clustering was performed on the Harmony-corrected data, with a resolution of 1.2, and defined major clusters composed of epithelial cells, B and plasma cells, T and NK cells, dendritic cells, monocytes, macrophages and neutrophils. The 50,610 myeloid cells were reintegrated and reclustered.

\section{Data analysis and statistics}

All statistical analyses were performed with R (v.3.6.1). The ggpubr (v.0.2.5) and ImerTest (v.3.1.2) packages were used for statistical tests. The gtsummary package (v.1.3.0) was used for the table containing clinical information (Supplementary Table 1). The sva package (v.3.32.1) was used to correct the batch effect of RNA-seq. Packages ggplot2 (v.3.2.1) and pheatmap (v.1.0.12) were used for the graphical representations of RNA-seq analyses. The Seurat package (v.3.1.0) was used for all analyses of single-cell RNA-seq. For the comparison of groups at time point $t_{0}$, $P$ values were obtained for two-tailed Wilcoxon rank-sum tests. For longitudinal analysis in the pneumonia group, the $P$ values for comparisons of $t_{1}$ and $t_{0}$ were obtained using two-tailed Wilcoxon signed-rank tests. No statistical tests were performed for $t_{2}$ in this group. For the ARDS group, a mixed model was computed, with time point as a fixed effect (categorical variable) and patient as a random effect. Confidence intervals and $P$ values were based on the $t$-distribution, with degrees of freedom according to the Kenward-Roger method, and the normality of residuals was verified. Plots were drawn with GraphPad Prism v.8.1.1. Box plots show the median (centre line) and 25 th to 75 th percentiles (box) and the whiskers denote the maximum and minimum values.

\section{Reporting summary}

Further information on research design is available in the Nature Research Reporting Summary linked to this paper.

\section{Data availability}

The RNA-seq data for two BALF samples from patients (each in duplicate), three PBMC samples from healthy control individuals and three PBMC samples from patients with COVID-19 were downloaded from the National Genomics Data Center (https://bigd.big.ac.cn/; accession number PRJCA002326). The RNA-seq data for three BALF samples from healthy control individuals were downloaded from the SRA database (accession numbers SRR10571724, SRR10571730 and SRR10571732). The single-cell RNA-seq data are available from the Gene Expression Omnibus (GEO) database (accession number GSE145926). Additional materials or data are available from the corresponding author upon reasonable request.

31. Ai, T. et al. Correlation of chest CT and RT-PCR testing for coronavirus disease 2019 (COVID-19) in China: a report of 1014 cases. Radiology 296, E32-E40 (2020).

32. Amrane, S. et al. Rapid viral diagnosis and ambulatory management of suspected COVID-19 cases presenting at the infectious diseases referral hospital in Marseille, France, - January 31st to March 1st, 2020: a respiratory virus snapshot. Travel Med. Infect. Dis. https://doi.org/10.1016/j.tmaid.2020.101632 (2020).

33. Nurtop, E. et al. Combination of ELISA screening and seroneutralisation tests to expedite Zika virus seroprevalence studies. Virol. J. 15, 192 (2018).

34. Xiong, Y. et al. Transcriptomic characteristics of bronchoalveolar lavage fluid and peripheral blood mononuclear cells in COVID-19 patients. Emerg. Microbes Infect. 9, 761-770 (2020).

35. Dobin, A. et al. STAR: ultrafast universal RNA-seq aligner. Bioinformatics 29, 15-21 (2013).

36. Liao, Y., Smyth, G. K. \& Shi, W. featureCounts: an efficient general purpose program for assigning sequence reads to genomic features. Bioinformatics 30, 923-930 (2014).

37. Johnson, W. E., Li, C. \& Rabinovic, A. Adjusting batch effects in microarray expression data using empirical Bayes methods. Biostatistics 8, 118-127 (2007).

38. Love, M. I., Huber, W. \& Anders, S. Moderated estimation of fold change and dispersion for RNA-seq data with DESeq2. Genome Biol. 15, 550 (2014).

39. Korsunsky, I. et al. Fast, sensitive and accurate integration of single-cell data with Harmony. Nat. Methods 16, 1289-1296 (2019).

Acknowledgements We thank all of the healthcare workers involved in the analysis, diagnosis and treatment of patients at AP-HM and Hôpital Laveran, especially Elise Kaspi, Eric Garnotel, Corinne Surcouf, Francois Xavier Le Flem (Bataillon des Marins Pompiers Marseille). We thank all our patients, supporters and families for their confidence in our work. The E.V. laboratory at CIML and Assistance-Publique des Hôpitaux de Marseille is supported by funding from the European Research Council (ERC) under the European Union's Horizon 2020 research and innovation program (TILC, grant agreement number 694502 and MInfla-TILC, grant agreement number 875102 - MInfla-Tilc), the Agence Nationale de la Recherche including the PIONEER Project (ANR-17-RHUS-0007), MSDAvenir, Innate Pharma and institutional grants awarded to the CIML (INSERM, CNRS, and Aix-Marseille University) and Marseille Immunopole.

Author contributions J.C., F.V. and E.V. initiated and designed the research. O.D. and E.V. wrote the manuscript with the help of other co-authors. O.D., F.V., L.B., J.F., S.C., M.-L.T., A.M., R.R., P.A., A. Represa, C.P., L.A.M., W.B., N.B., C.C., F. Caraguel, B. Carrette, F. Carrette, F. Chanuc, R.C., A.F., M. Giordano, M.G.-M., M.G.-P., N.G., G.G., F.G., G.H., S.J., J.L., M.L.V., N.L., M.M., E.B., A.S., A. Reboul, E.M., C.N.-C., V.P., P.R., L.S., J.-B.V., M.V., Y.M., R.Z., L.L., J.G., M. Etiennot, S.U., B.P., B. Coutard, C.D. and L.D. performed the experiments and analysed and/or interpreted results. J.C., N.C.B., P.Y.C., E.L.D., C.G., P.S., M. Gainnier, M. Ebbo, N.S., J.-M.F., L.P., L.V., B.A., A.B., B.F., E.J., J.S., V.V., J.-R.H., B.P., C.D., L.D., J.-P.B., A.C., E.D., P.-O.V. and H.S. were in charge of patient care and contributed to the discussion of the results. L.B., N.C.B., J.F., S.C., M.-L.T. and A.M. contributed equally.

Competing interests O.D., L.B., J.F., S.C., M.-L.T., A.M., R.R., P.A., A.Represa, L.A.M., W.B., N.B., C.C., F. Caraguel, B.Carrette, F.Carrette, F.Chanuc, R.C., A.F., M.Giordano, M.G.-M., M.G.-P., N.G., G.G., F.G., G.H., S.J., J.L., M.L.V., N.L., M.M., E.B., A.S., A.Reboul, E.M., C.N.-C., V.P., P.R., L.S., J.-B.V., M.V., R.Z., Y.M. and E.V. are employees of Innate Pharma. The other authors declare no competing interests.

Additional information

Supplementary information is available for this paper at https://doi.org/10.1038/s41586-0202600-6.

Correspondence and requests for materials should be addressed to E.V.

Peer review information Nature thanks Bart Lambrecht and the other, anonymous, reviewer(s) for their contribution to the peer review of this work.

Reprints and permissions information is available at http://www.nature.com/reprints. 
a

\begin{tabular}{|c|c|c|c|c|}
\hline Status & $\mathrm{HC}$ & Pauci & Pneumonia & ARDS \\
\hline \multirow[b]{3}{*}{ Status } & 9 & 5 & 6 & 0 \\
\hline & 0 & 5 & 22 & 26 \\
\hline & $\mathrm{HC}$ & Pauci & Pneumonia & ARDS \\
\hline \multirow{2}{*}{$\begin{array}{l}\text { Non-neutralizing } \\
\text { Neutralizing }\end{array}$} & NA & 0 & 1 & 0 \\
\hline & NA & 1 & 18 & 21 \\
\hline
\end{tabular}

C b
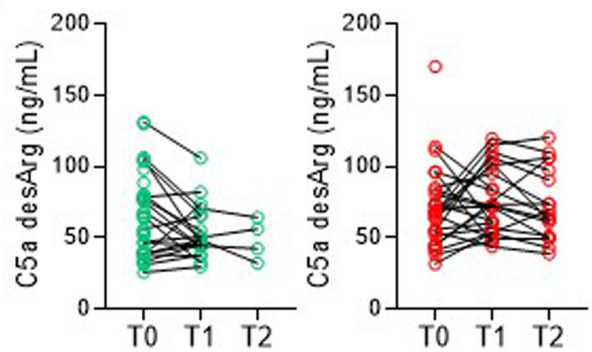
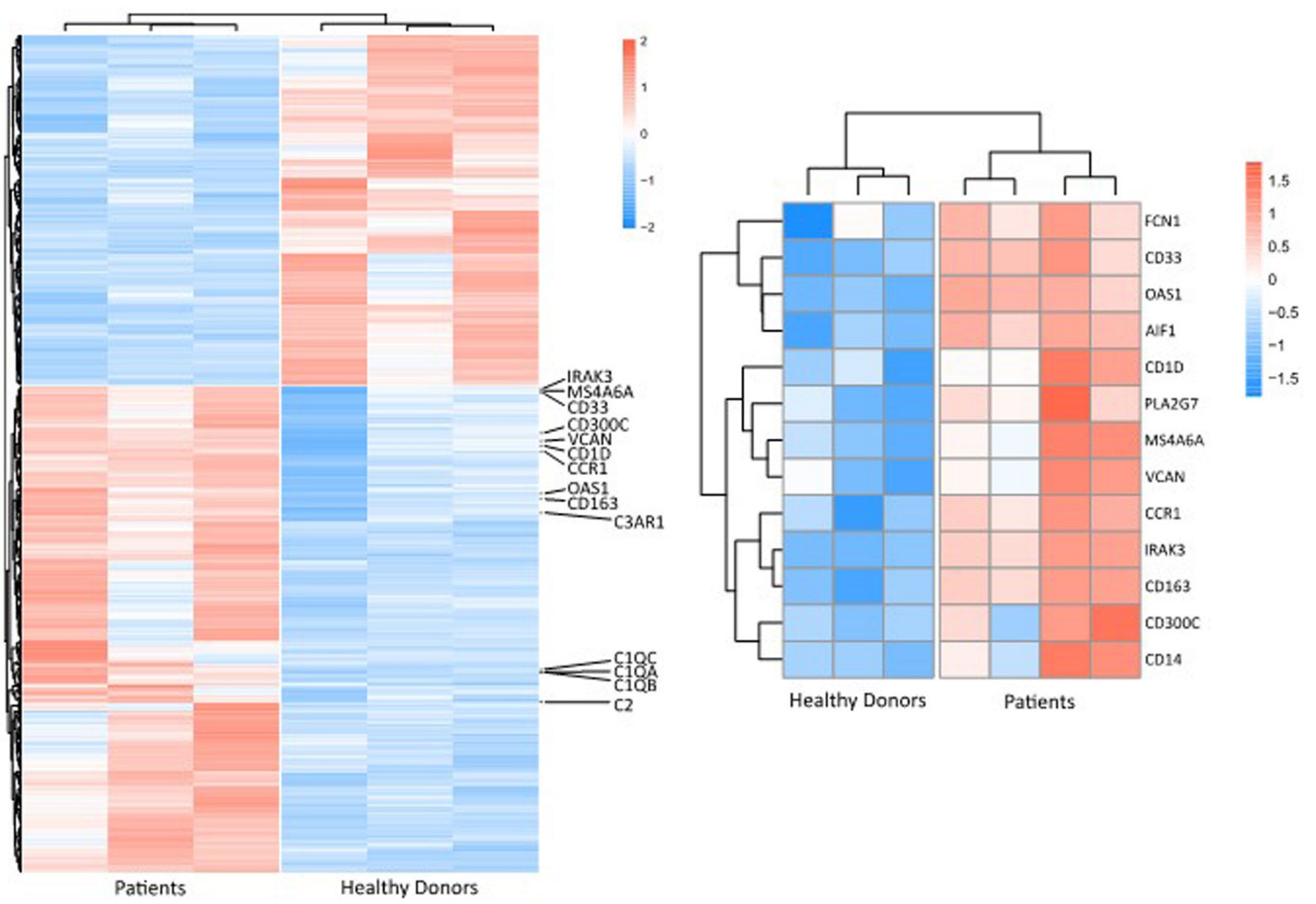

d

$H \& E$

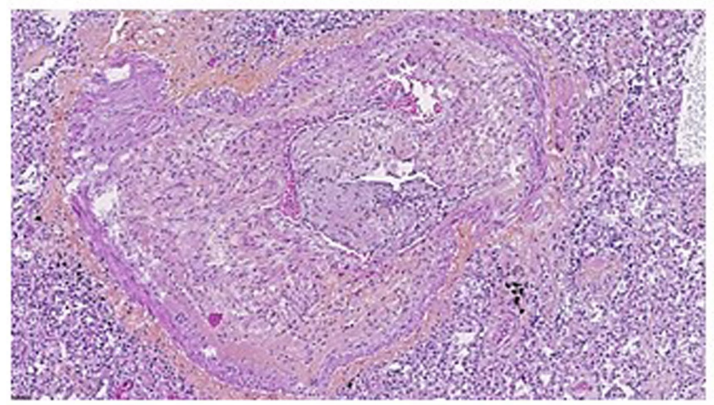

C5b9

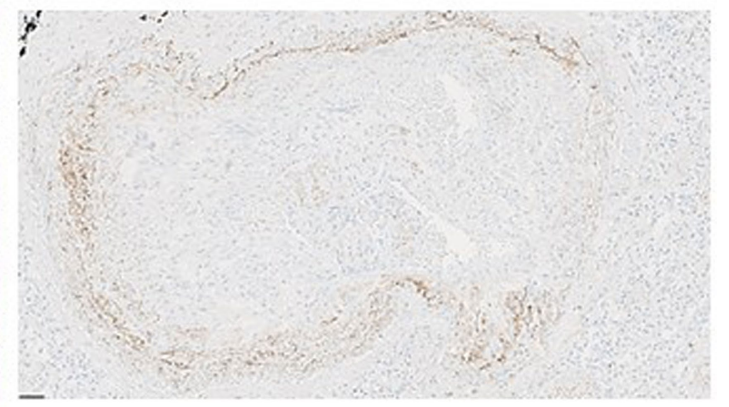

intervals and $P$ values are based on the $t$-distribution, with degrees of freedom according to the Kenward-Roger method (not significant). Each symbol represents a single donor.c, Left, Heat map of genes that are differentially expressed (log-transformed fold change $>2$ and false-discovery rate $<5 \%$ ) between PBMC samples from healthy control individuals and patients with COVID-19. Right, Heat map of monocyte and macrophage metagene expression in lung samples from healthy control individuals and patients with COVID-19. d, Three lung samples from patients who are deceased were obtained and were suitable for IHC analysis. Left, H\&E staining of obliterating endarteritis lesions in the lungs of a representative patient with COVID-19. Right, C5b9 IHC staining on lung sections of a representative patient with COVID-19, demonstrating complement pathway activation in the lung. Scale bars, $50 \mu \mathrm{m}$. model was computed with time point (categorical variable) as a fixed effect and patient as a random effect. $n=28$ for $t_{0}, n=23$ for $t_{1}$ and $n=18$ for $t_{2}$. Confidence
Extended Data Fig. 1 | Immune activation in patients with COVID-19.

a, Number of patients for each level of disease severity, classified according $t_{0}\left(<72 \mathrm{~h}\right.$ after the start of hospital care) and $t_{1}$ (between days 5 and 10 ).

b. Concentration of $\mathrm{C} 5$ a desArg in plasma from patients with pneumonia beginning of hospital care; $t_{1}$, between days 5 and $10 ; t_{2}$, after day $10 . P$ values for the comparison of $t_{1}(n=19)$ and $t_{0}(n=34)$ in the pneumonia group were obtained using two-tailed Wilcoxon signed-rank tests (not significant). No statistical tests were performed for $t_{2}(n=4)$. For the ARDS group, a mixed 

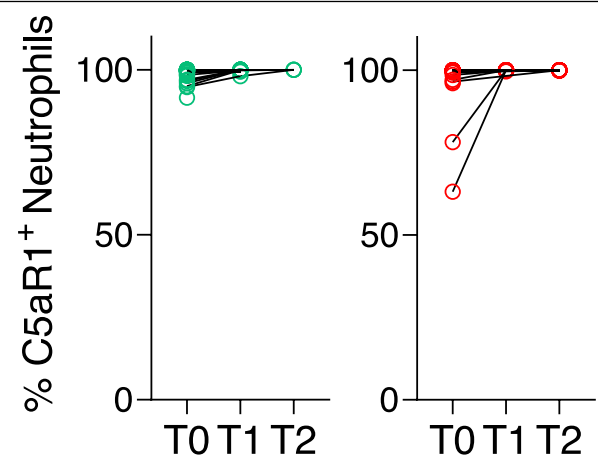

Extended Data Fig. 2 |C5aR1 expression remained stable on myeloid cells during the course of COVID-19. The percentage of C5aR1-expressing neutrophils and monocytes in patients with pneumonia (green) and ARDS (red) followed over time. $t_{0},<72 \mathrm{~h}$ after the start of hospital care; $t_{1}$, between days 5

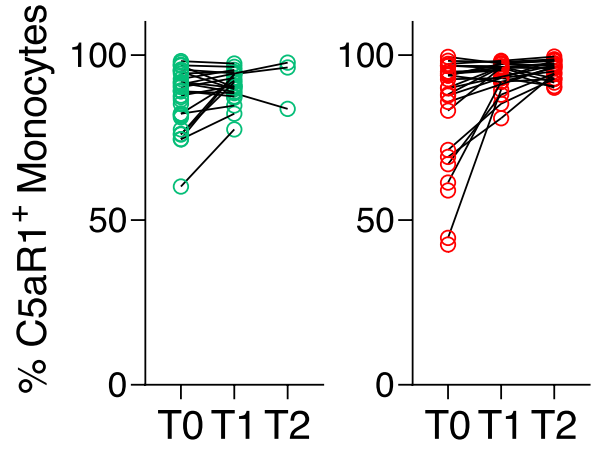

and $10 ; t_{2}$, after day 10. For the pneumonia group, $n=34$ for $t_{0}, n=18$ (neutrophils) and 21 (monocytes) for $t_{1}$ and $n=3$ for $t_{2}$. For the ARDS group, $n=28$ for $t_{0}, n=23$ for $t_{1}$ and $n=22$ for $t_{2}$. Each symbol represents a single donor. 

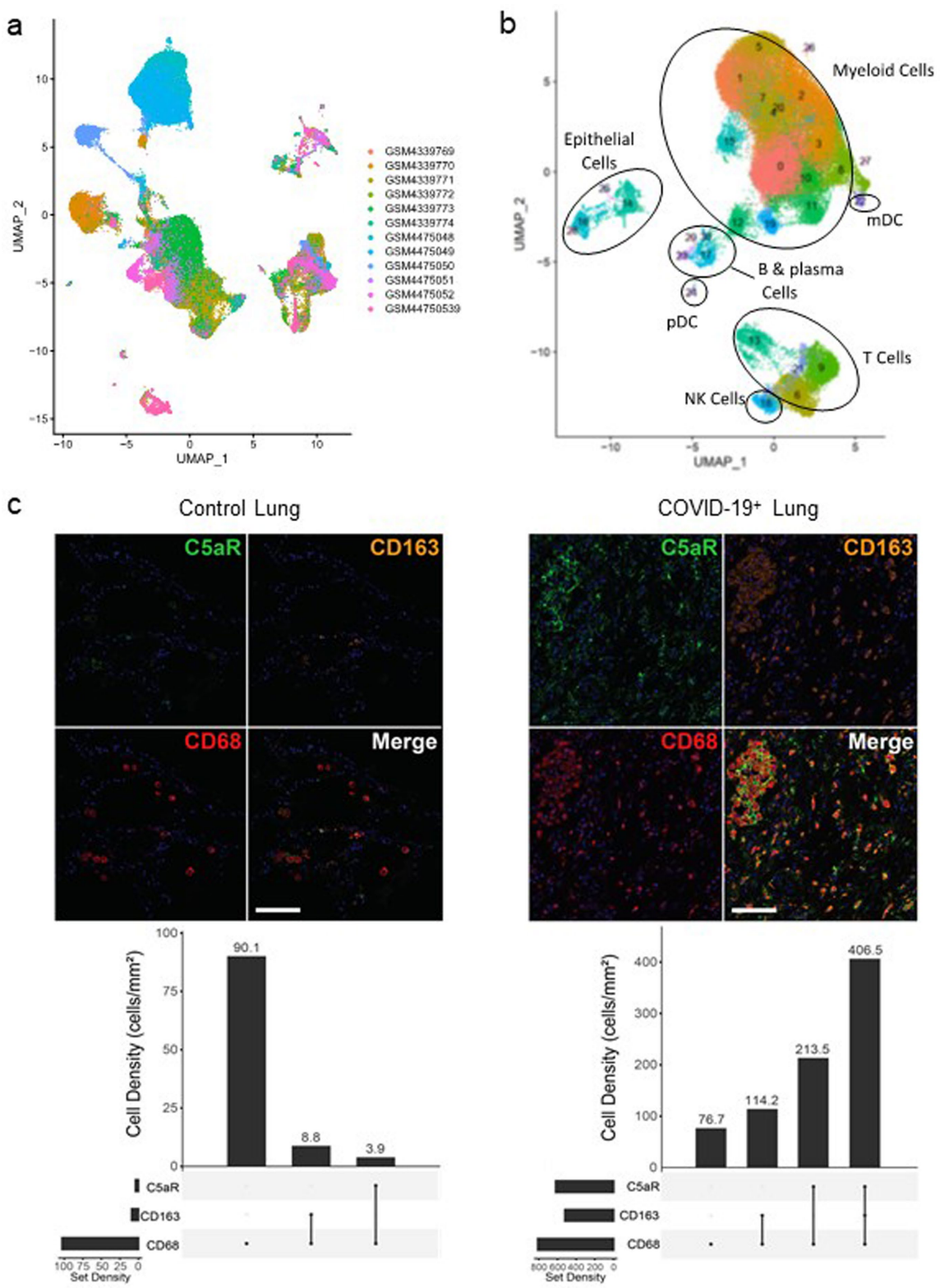

d

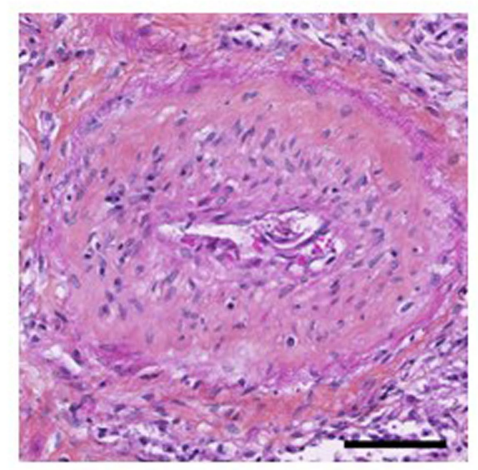

Extended Data Fig. 3 | Myeloid cell analysis in patients with COVID-19. $\mathbf{a}, \mathbf{b}$, Integration of transcriptomic single-cell data with Harmony. a, UMAP projection of donors before integration. $\mathbf{b}$, UMAP projection of major cell types and associated clusters after integration by Harmony. mDC, myeloid dendritic cells. c, Representative multiplexed IHC staining of C5aR (green), CD68 (red) and CD163 (orange) on lung sections from a control individual or patients with COVID-19 among three samples from patients with COVID-19 who are deceased and for whom tissue was available for IHC analyses. Scale bars, $100 \mu \mathrm{m}$. Quantifications show the cell density per $\mathrm{mm}^{2}$ of multiplexed IHC

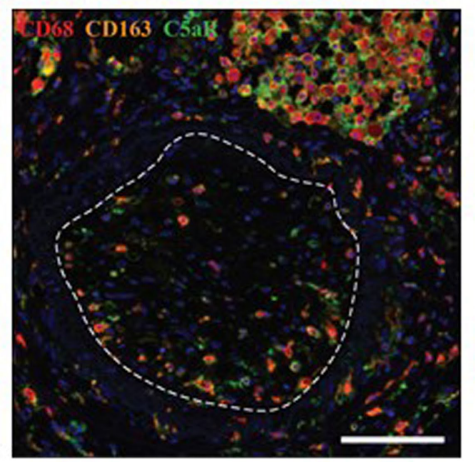

staining of C5aR1, CD68 and CD163. d, Three samples from patients who are deceased were obtained and were suitable for IHC analysis. Endoarteritis lesions were observed in two out of three patients, consistent with previous reports. The patient without endoarteritis lesions did not die from COVID-19. Left, representative $\mathrm{H} \& \mathrm{E}$ staining of obliterating endarteritis lesions in the lungs of patients with COVID-19. Right, representative multiplexed IHC staining of C5aR1 (green), CD68 (red) and CD163 (orange) showing that obliterating endarteritis was frequently associated with $\mathrm{C} \mathrm{aR} 1^{+}$macrophages surrounding the arteries and in the thrombus (white dotted line). Scale bars, $100 \mu \mathrm{m}$. 

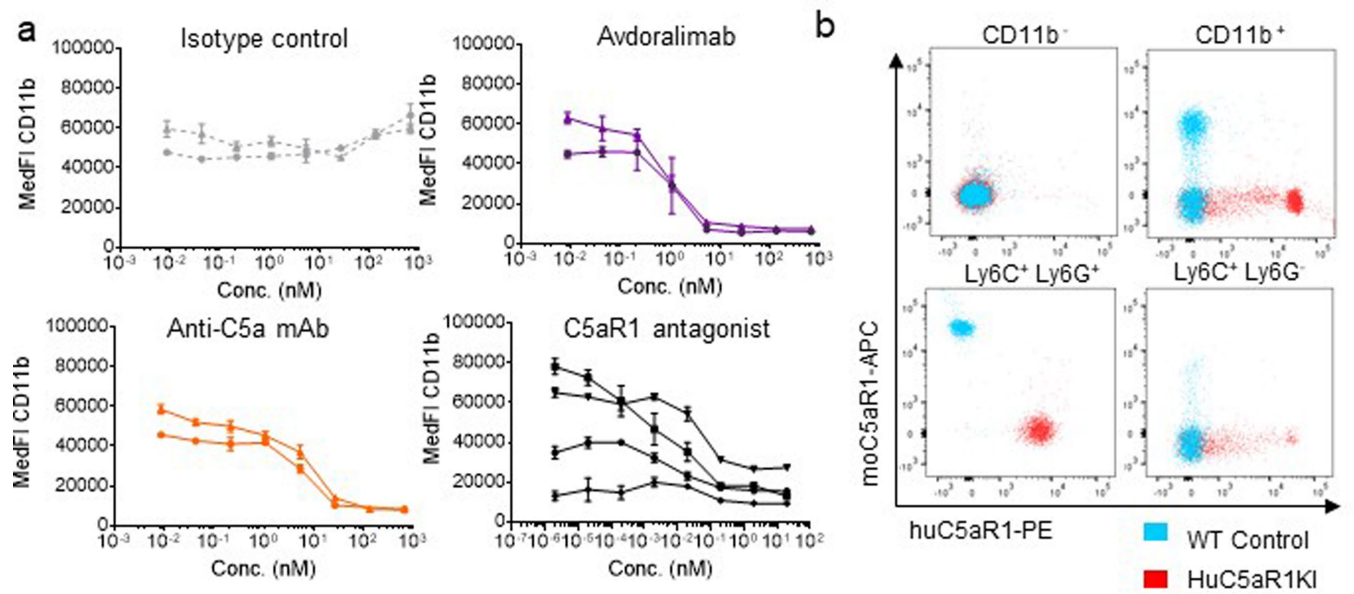

C
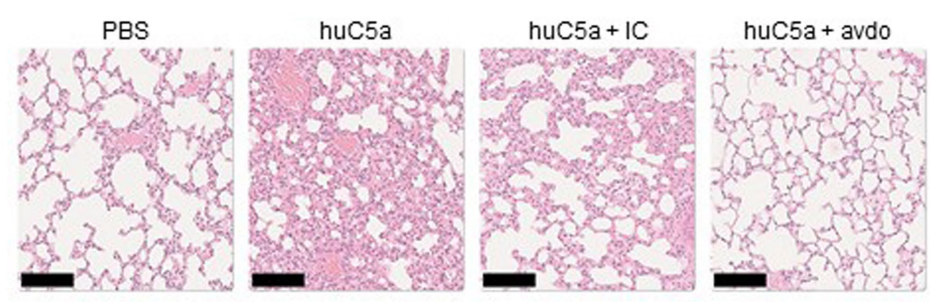

d

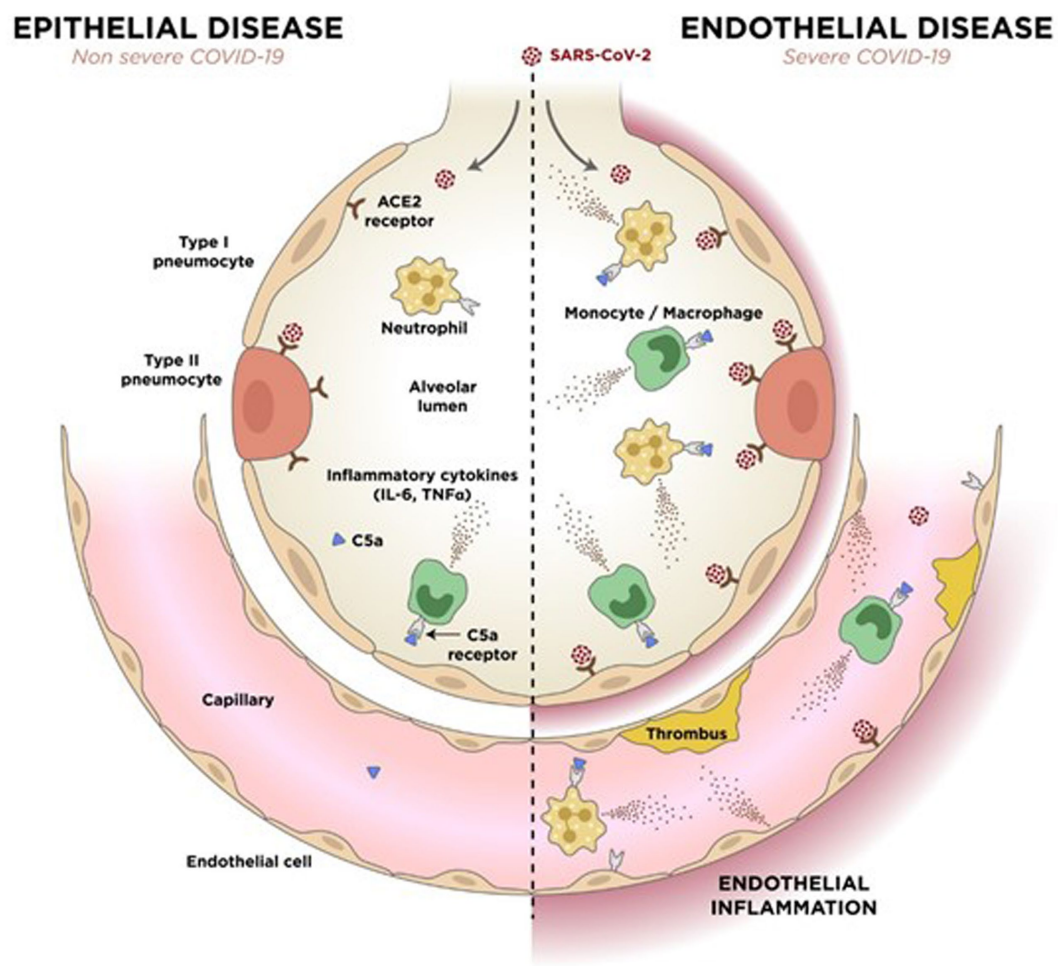

Extended Data Fig. 4 | Targeting C5aR1 to block C5a-mediated activation of myeloid cells. a, Analysis of the efficacy of increasing doses of avdoralimab (purple), an anti-C5a monoclonal antibody (orange), a C5aR1 antagonist (avacopan, black) or isotype control (grey) to block C5a-induced upregulation of CD11b on human neutrophils. Each line represents the mean \pm s.d. of the median fluorescence index from a single donor from experimental duplicates (isotype control) or triplicates (inhibitors). b, Comparative expression of mouse C5aR1 (moC5aR1) and human C5aR1 (huC5aR1) on $\mathrm{CD}^{1} 1 \mathrm{~b}^{-}$non-myeloid cells, CD11b ${ }^{+}$myeloid cells, including $\mathrm{Ly}_{6 \mathrm{C}} \mathrm{Cy}^{+} \mathrm{G}^{+}$neutrophils and $\mathrm{Ly}^{-} \mathrm{C}^{+} \mathrm{Ly} 6 \mathrm{G}^{-}$ monocytes, from wild-type (blue) and HsC5AR1 knock-in (red) mice.c, $\mathrm{H} \& \mathrm{E}$ staining of lungs from $\mathrm{HsC} 5 A R 1$ knock-in mice treated intranasally with $\mathrm{HsC} 5 \mathrm{a}$. Mice were pretreated with avdoralimab (avdo) or isotype control (IC), where indicated. Scale bar, $100 \mu \mathrm{m}$. Images are representative of two independent experiments. d, A model of C5a involvement in COVID-19:SARS-CoV-2 infects the human airway epithelium through the ACE2 receptors that are located predominantly on type-II pneumocytes. Left, in non-severe COVID-19, the infection remains confined to the epithelium (epithelial disease), because of the efficient action of the immune system. C5a enables the recruitment of myeloid cells without triggering an inflammatory storm and the virus is eliminated. Right, in severe COVID-19, SARS-CoV-2 escapes the immune system, crosses the epithelium and infect endothelial cells (endothelial disease). The myeloid cells recruited by $\mathrm{C} 5 \mathrm{a}$ and endothelial cells release large amounts of inflammatory cytokines. COVID-19-related cytokine storm and endothelialitisassociated microthrombosis are triggered. The condition of the patient worsens and the virus can infect other organs. 


\section{natureresearch}

Corresponding author(s): Prof Eric Vivier

Last updated by author(s): Jul 15, 2020

\section{Reporting Summary}

Nature Research wishes to improve the reproducibility of the work that we publish. This form provides structure for consistency and transparency in reporting. For further information on Nature Research policies, see Authors \& Referees Editorial Policy Checklist

\section{Statistics}

For all statistical analyses, confirm that the following items are present in the figure legend, table legend, main text, or Methods section.

n/a Confirmed

$\square \bigotimes$ The exact sample size $(n$

$\square$ \A statement on whether measurements were taken from distinct samples or whether the same sample was measured repeatedly

The statistical test(s) used AND whether they are one- or two-sided

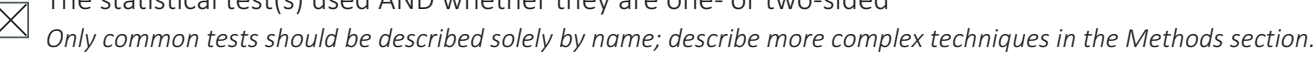

\ $\square$ A description of all covariates tested

$\square$ A description of any assumptions or corrections, such as tests of normality and adjustment for multiple comparisons

$\square$ A full description of the statistical parameters including central tendency (e.g. means) or other basic estimates (e.g. regression coefficient)

$\triangle$ AND variation (e.g. standard deviation) or associated estimates of uncertainty (e.g. confidence intervals)

$\varnothing$ For null hypothesis testing, the test statistic (e.g. F $t \quad r \quad P$

$\triangle$ Give $P$ values as exact values whenever suitable.

Х $\square$ For Bayesian analysis, information on the choice of priors and Markov chain Monte Carlo settings

Х For hierarchical and complex designs, identification of the appropriate level for tests and full reporting of outcomes

$\bigotimes \square$ Estimates of effect sizes (e.g. Cohen's $d r$

Our web collection on statistics for biologists

\section{Software and code}

Policy information about availability of computer code

Data collection $\quad$ RNASeq pipeline: The reads were mapped to human genome (hg38) release 96 from Ensembl with STAR47. PCR replicates mapping to the human genome were removed with the Picard MarkDuplicates program (Broad Institute 2019, http://broadinstitute.github.io/ picard/). Gene expression was calculated with featureCounts function in the SubReads package (v1.6.4)48. TPM (transcripts per million) values were calculated from the raw counts and log2-transformed.

Enspire system and software (Perkin Elmer) version 4.13.3005.1482 were used to assess the C5a-desarg concentrations

Meso Quickplex SQ 120 system and MSD Workbench 3.0.18 software (MesoScale Discovery) were used to follow the cytokines concentrations.

Data analysis
All statistics analyses were performed using R (version 3.6.1) and GraphPad Prism (version 8.1.1)
Packages ggpubr (version 0.2.5) and ImerTest (version 3.1.2) were used for statistical tests and gtsummary (version 1.3.0) for clinical table.
Package sva (version 3.32.1) was used to correct the batch effect of RNAseq. Package DESeq2 (version 1.24.0) was used for the differential analysis. Packages ggplot2(version 3.2.1) and pheatmap (1.0.12) were used for the graphical representations of RNASeq analyses in the supplementary figures.
Package Seurat (version 3.1.0) was used for all analyses of single-cell RNASeq
Graphical representations were performed using GraphPad Prism (version 8.1.1)
The FCS3.0 files obtained were exported from BD FACSDiva software version 8.0 and imported into FlowJo v.10.5.2 (BD Biosciences) CytoNuclear FL module from Halo software (Indica Labs) version 3.0.311 was used to count the cells that had crossed the membrane for the neutrophil migration assay
All analyses of digital pathology were performed with Halo (Indica Labs) version 3.0.311 and R version 3.6.1. 
Policy information about availability of data

All manuscripts must include a data availability statement

- Accession codes, unique identifiers, or web links for publicly available datasets

- A list of figures that have associated raw data

- A description of any restrictions on data availability

PRICA002326 from https://bigd.big.ac.cn/. SRR10571724, SRR10571730, and SRR10571732 from from the SRA database

GSE145926 from the Gene Expression Omnibus (GEO) database (http://www. https://www.ncbi.nlm.nih.gov/geo)

\section{Field-specific reporting}

Please select the one below that is the best fit for your research. If you are not sure, read the appropriate sections before making your selection.

$\bigotimes$ Life sciences $\quad \square$ Behavioural \& social sciences $\quad \square$ Ecological, evolutionary \& environmental sciences

For a reference copy of the document with all sections, see nature.com/documents/nr-reporting-summary-flat.pdf

\section{Life sciences study design}

All studies must disclose on these points even when the disclosure is negative.

Sample size

As COVID-19 is a new pandemic acute respiratory disease and differences between groups unknown, it was not suitable to estimate the sample size with expected differences. However, we anticipated bigger differences between Pneumo \& SARS patients compared to Healthy or Pauci than between the two most severe groups.

We decided, thus, to include $10 \mathrm{HC}, 10$ Pauci, 30-40 Pneumonia and 30-40 SARS patients to be able to detect by t-test a difference of:

- One standard deviation (sd) with a power of $80 \%$ at a significance level of $5 \%$ between HC or Pauci with Pneumo or SARS

- 0.6-0.7 sd with a power of $80 \%$ at a significance level of $5 \%$ between Pneumo and SARS.

For in vitro experiments with cells from COVID19 patients: With a sample size of cells isolated from frozen PBMCs of 10 COVID-19 patients, we are able to detect by a paired-t-test a difference of one standard deviation (sd) with a power of $80 \%$ at a significance level of $5 \%$. To be sure to get results despite possible technical problems, we included 15 samples of cells isolated from frozen PBMC.

For in-vivo mouse experiments: Based on literature, we decided to include 7 mice per group. Using the mean and standard deviation observed in the experiment, we calculated a posteriori the sample size required to achieve a power of $90 \%$ at a significance level of $5 \%$.

The minimum sample size required to achieve a power of $90 \%$ was 6 mice per group.

Data exclusions

Cytometry data were excluded when cell number of population of interest were under 200 events.

For In-vitro experiments with patient samples, in the case of not enough cells were harvested to perform the all experimental design, some conditions may lack. One patient was also excluded due to a technical problem.

Replication

Soluble factors analysis and immune cell phenotyping by FACS were performed on a large number of patients (34 pneumonia, 28 ARDS, 10 Paucisymptomatic and 10 healthy controls) to validate reproductibilty of findings BALF analysis were performed from 4 different COVID-19 patients.

Three samples from deceased patients suitable for IHC analyses were obtained and processed for H\&E and multiplexed IHC staining In vitro experiment of stimulation of COVID-19 monocytes with TLR agonists + C5a +/- avdoralimab was repeated twice and performed on cells from a total of 15 samples from COVID-19 patients and 5 healthy donors.

Efficacy of avdoralimab to block CD11b upregulation on neurophils from 3 healthy donors was performed in two independent experiments. Avdoralimab blockade of C5a induced ALl experiment was repeated twice wich 7 mice per group. IHC analysis on lungs from mice upon C5a induced ALI experiment + -- avdoralimab was repeated twice.

Randomization Human samples were included in groups based on the severity (paucisymptomatic, pneumonia or ARDS) of the patient when recruited. For in vitro experiments, patient samples were selected for high percentages of monocytes in frozen PBMC to allow recovery of enough cells to perform the experiments.

As no control was possible untill the end of the experimental procedures, no randomization was performed for in vivo experiments in mice.

Blinding

The goal of our study was to investigate the immune response in COVID-19 patients occuring at different severity stage of the disease. Blinding was not possible as we needed to organize our cohort in 3 different groups. 


\section{Behavioural \& social sciences study design}

All studies must disclose on these points even when the disclosure is negative.

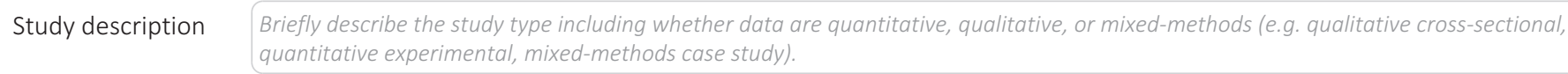

Research sample State the research sample (e.g. Harvard university undergraduates, villagers in rural India) and provide relevant demographic information (e.g. age, sex) and indicate whether the sample is representative. Provide a rationale for the study sample chosen. For studies involving existing datasets, please describe the dataset and source.

Sampling strategy Describe the sampling procedure (e.g. random, snowball, stratified, convenience). Describe the statistical methods that were used to predetermine sample size OR if no sample-size calculation was performed, describe how sample sizes were chosen and provide a rationale for why these sample sizes are sufficient. For qualitative data, please indicate whether data saturation was considered, and what criteria were used to decide that no further sampling was needed.

Data collection Provide details about the data collection procedure, including the instruments or devices used to record the data (e.g. pen and paper, computer, eye tracker, video or audio equipment) whether anyone was present besides the participant(s) and the researcher, and whether the researcher was blind to experimental condition and/or the study hypothesis during data collection.

Timing

Indicate the start and stop dates of data collection. If there is a gap between collection periods, state the dates for each sample cohort.

Data exclusions

If no data were excluded from the analyses, state so OR if data were excluded, provide the exact number of exclusions and the rationale behind them, indicating whether exclusion criteria were pre-established.

Non-participation State how many participants dropped out/declined participation and the reason(s) given OR provide response rate OR state that no participants dropped out/declined participation.

Randomization If participants were not allocated into experimental groups, state so OR describe how participants were allocated to groups, and if allocation was not random, describe how covariates were controlled.

\section{Ecological, evolutionary \& environmental sciences study design}

All studies must disclose on these points even when the disclosure is negative.

Study description

Research sample

Sampling strategy

Data collection

Timing and spatial scale

Data exclusions

Reproducibility

Randomization

Blinding
Briefly describe the study. For quantitative data include treatment factors and interactions, design structure (e.g. factorial, nested, hierarchical), nature and number of experimental units and replicates.

Describe the research sample (e.g. a group of tagged Passer domesticus, all Stenocereus thurberi within Organ Pipe Cactus National Monument), and provide a rationale for the sample choice. When relevant, describe the organism taxa, source, sex, age range and any manipulations. State what population the sample is meant to represent when applicable. For studies involving existing datasets, describe the data and its source.

Note the sampling procedure. Describe the statistical methods that were used to predetermine sample size OR if no sample-size calculation was performed, describe how sample sizes were chosen and provide a rationale for why these sample sizes are sufficient.

Describe the data collection procedure, including who recorded the data and how.

Indicate the start and stop dates of data collection, noting the frequency and periodicity of sampling and providing a rationale for these choices. If there is a gap between collection periods, state the dates for each sample cohort. Specify the spatial scale from which the data are taken

If no data were excluded from the analyses, state so OR if data were excluded, describe the exclusions and the rationale behind them, indicating whether exclusion criteria were pre-established.

Describe the measures taken to verify the reproducibility of experimental findings. For each experiment, note whether any attempts to repeat the experiment failed OR state that all attempts to repeat the experiment were successful.

Describe how samples/organisms/participants were allocated into groups. If allocation was not random, describe how covariates were controlled. If this is not relevant to your study, explain why.

Did the study involve field work? $\square$ Yes $\square$ No 
Field conditions

Describe the study conditions for field work, providing relevant parameters (e.g. temperature, rainfall).

Location

Access and import/export

Describe the efforts you have made to access habitats and to collect and import/export your samples in a responsible manner and in compliance with local, national and international laws, noting any permits that were obtained (give the name of the issuing authority, the date of issue, and any identifying information).

Disturbance

\section{Reporting for specific materials, systems and methods}

We require information from authors about some types of materials, experimental systems and methods used in many studies. Here, indicate whether each material, system or method listed is relevant to your study. If you are not sure if a list item applies to your research, read the appropriate section before selecting a response.

Materials \& experimental systems

\begin{tabular}{l|l}
\multicolumn{2}{l}{ Methods } \\
\hline n/a & Involved in the study \\
$\square$ & $\square$ ChIP-seq \\
$\square$ & $\bigotimes$ Flow cytometry \\
$\square$ & $\square$ MRI-based neuroimaging
\end{tabular}

\begin{tabular}{l|l}
\hline$n / a$ & Involved in the study \\
$\square$ & $\square$ Antibodies \\
$\square$ & $\square$ Eukaryotic cell lines \\
$\square$ & $\square$ Animals and other organisms \\
$\square$ & $\bigotimes$ Human research participants \\
$\square$ & $\square$ Clinical data
\end{tabular}

\section{Antibodies}

Antibodies used

Validation

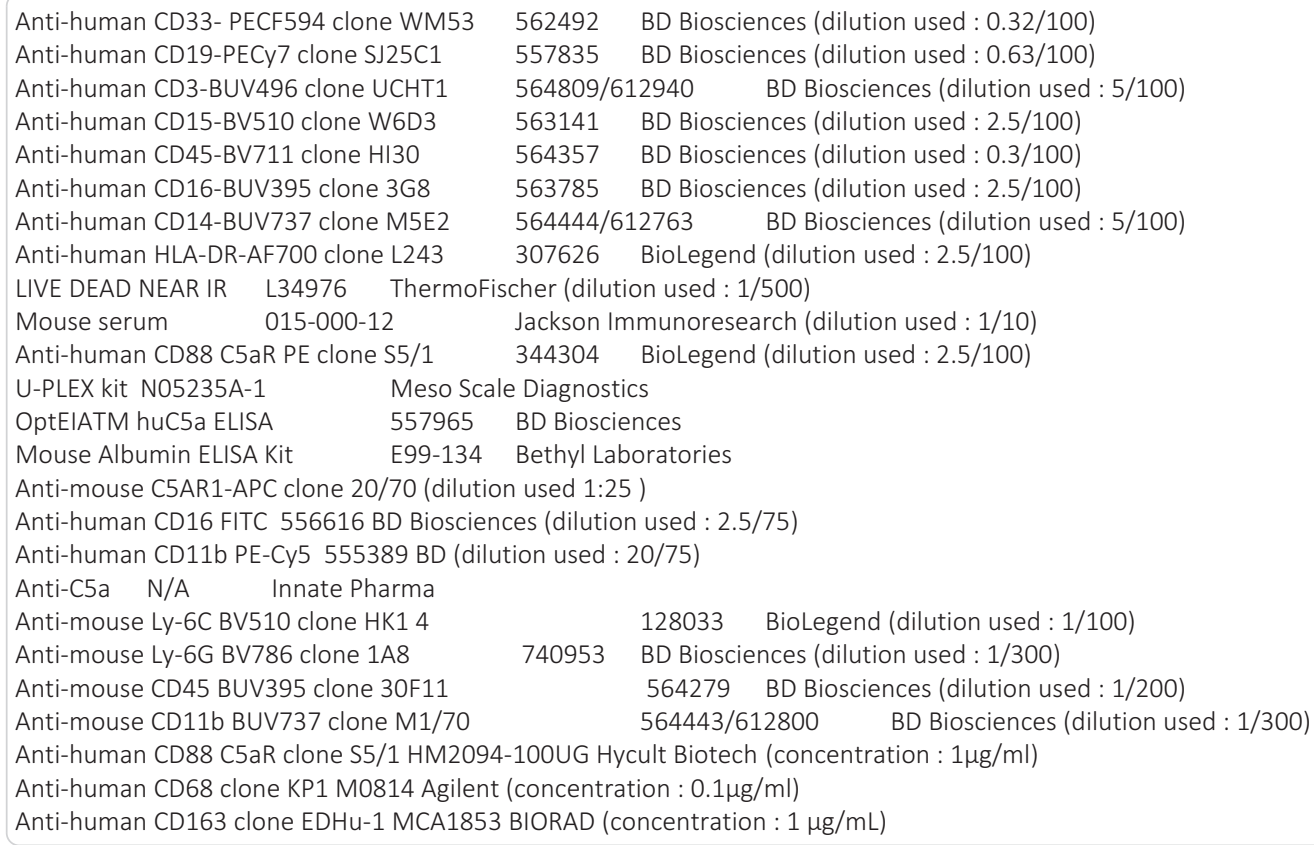

For Flow cytometry : Titration of each antibodies have been performed under the same conditions as the study (whole blood, $4^{\circ}$ $\mathrm{C}$, incubation 30 minutes). The various panels have been tested many times on human (healthy donors and cancer patients) and mouse samples and have been recorded as templates in order to follow a standardization process on BD instrument. For IHC: Primary antibody specificity was validated on formalin-fixed paraffin-embedded cell pellets and tissues. For C5aR, human C5aR-transfected and untransfected cells and kidney tissue were used. For CD68, THP-1 and JURKAT cell lines as well as liver tissue were tested. Finally, SU-DHL-1 and THP-1 cell lines and liver tissue were used for CD163 staining validation. 


\section{Eukaryotic cell lines}

Policy information about cell lines

Cell line source(s)

State the source of each cell line used.

Authentication

Describe the authentication procedures for each cell line used OR declare that none of the cell lines used were authenticated.

Mycoplasma contamination

Confirm that all cell lines tested negative for mycoplasma contamination OR describe the results of the testing for

mycoplasma contamination OR declare that the cell lines were not tested for mycoplasma contamination.

Commonly misidentified lines Name any commonly misidentified cell lines used in the study and provide a rationale for their use.

(See ICLAC

\section{Palaeontology}

Specimen provenance

Provide provenance information for specimens and describe permits that were obtained for the work (including the name of the issuing authority, the date of issue, and any identifying information).

Specimen deposition

Indicate where the specimens have been deposited to permit free access by other researchers.

Dating methods

If new dates are provided, describe how they were obtained (e.g. collection, storage, sample pretreatment and measurement). where they were obtained (i.e. lab name), the calibration program and the protocol for quality assurance OR state that no new dates are provided.

Tick this box to confirm that the raw and calibrated dates are available in the paper or in Supplementary Information.

\section{Animals and other organisms}

Policy information about studies involving animals $\underline{\text { ARRIVE guidelines }}$

Laboratory animals

C57BI/6J female mice were purchased at Janvier Labs and used between 8 to 12 weeks old. HuC5aR1 KI mice were bred at Charles River Laboratories under specific pathogen-free conditions. After reception, mice were housed in Innate Pharma animal facilities and had a SOPF (Specific and Opportunist Pathogen Free) health status.

Animals were housed in an air-conditioned building. The targeted conditions for animal room environment were as follows:

Temperature: $22^{\circ} \mathrm{C} \pm 2^{\circ} \mathrm{C}$

Relative humidity: $40-75 \%$

Air changes: $15-20$ air changes per hour. Both supply and exhaust air are HEPA-filtered.

Lighting cycle: 12 hours light/dark daily lighting cycle

Caging: Mice were housed as single-sex social groups, in $500 \mathrm{~cm} 2$ cages containing between two and five animals.

Diet: Rats Mice Hamsters breeding irradiated diet

Water: Tap water, sterilized in an autoclave for $20 \mathrm{~min}$ at $121^{\circ} \mathrm{C}$, was available ad libitum to each animal via a bottle with a sipper tube.

Acclimatization period: The study animals were acclimated to their housing environment for at least 4 days prior to entering the study.

Female and male between 7 and 12 weeks old were used for experiments.

Wild animals

Field-collected samples

Ethics oversight

\section{No Wild animals were used in our study.}

No field collected samples were used in the study.

All animal experiments were performed in accordance with the rules of the Innate Pharma ethics committee and were approved by the ministere de l'enseignement superieur, de la recherche et de l'innovation - France (APAFIS\#25418-2020051512242806 v2).

Note that full information on the approval of the study protocol must also be provided in the manuscript.

\section{Human research participants}

Policy information about studies involving human research participants

Population characteristics

We recruited 72 COVID-19 patients with 3 levels of severity: intensive care unit patients with COVID-19-related ARDS, medical unit patients with COVID-19 related pneumonia requiring oxygen support and paucisymptomatic patients. Cohort carateristics: median age 61 years old, female $26 \%$ and male 74\%, COVID-19 diagnosed by RT PCR, nasopharingeal sampling or Chest CT-Scan.

10 healthy volunteers were also recruited, median age 54 years old, 8 men and 2 women, according to the usual gender distribution of COVID-19. Negativity for SARS-COV-2 was evluated by SARS-CoV-2 RT-PCR and serology analysis. 

COVID-19-related-ARDS (P/F ratio < 300) (ARDS group), 34 patients required oxygen support at a rate of less than $5 \mathrm{~L} / \mathrm{min}$ for COVID-19-related pneumonia (pneumonia group). Ten patients had a paucisymptomatic form of COVID-19 compatible with outpatient care (paucisymptomatic group). COVID-19 was diagnosed on the basis of positive SARS-CoV-2 RT-PCR on nasopharyngeal samples and/or typical CT-scan findings. We also included 10 healthy volunteers (control group), with no fever or symptoms in the days before sampling and negative for SARS-CoV-2 RT-PCR. The healthy volunteers were recruited in our laboratory and medical units. They were not sick at the time of the samples with no fever, cough or flu syndrome. They didn't take any treatment at the time of sampling. All their COVID-19 serologies were negative. They signed an authorization to participate.

Ethics oversight

The study protocol was approved on 03-27-2020 by the Committee for the Protection of Persons Ile-de-France III - France (\#2020-A00757-32)

Note that full information on the approval of the study protocol must also be provided in the manuscript.

\section{Clinical data}

Policy information about clinical studies

All manuscripts should comply with the ICMJE guidelines for publication of clinical research CONSORT checklist

Clinical trial registration

N/A

Study protocol

All the patients (and/or initially their families) provided written informed consent before sampling and for the use of their clinical and biological data. The study protocol was approved on 03-27-2020 by the Committee for the Protection of Persons Ile-deFrance III - France (\#2020-A00757-32). The pathological examination used in this study was performed secondary to a medical autopsy following COVID-19 related-death, with family agreement and notified to the representative of the Commission on Data Processing and Freedom (MRO03 research).

Data collection

Biological samples were first collected within three days of diagnosis and the start of care (TO: $<72 \mathrm{~h}$, early time-point). When possible, the next two time-points for sample collection were located between days 5 and 10 (T1: D5 to D10, intermediate timepoint) and after day 10 (T2: > D10, late time-point). Flow cytometry analyses were performed on fresh blood samples (EDTA tubes) and BALFs, immediately after collection

Outcomes

N/A

\section{ChIP-seq}

\section{Data deposition}

$\square$ Confirm that both raw and final processed data have been deposited in a public database such as GEO

Confirm that you have deposited or provided access to graph files (e.g. BED files) for the called peaks.

Data access links

May remain private before publication.

Files in database submission

Genome browser session (e.g. UCSC

\section{Methodology}

Replicates

Sequencing depth

Antibodies

Peak calling parameters

Data quality

Software
For "Initial submission" or "Revised version" documents, provide reviewer access links. For your "Final submission" document, provide a link to the deposited data.

Provide a list of all files available in the database submission.

Provide a link to an anonymized genome browser session for "Initial submission" and "Revised version" documents only, to enable peer review. Write "no longer applicable" for "Final submission" documents. 


\section{Plots}

Confirm that:

$\bigotimes$ The axis labels state the marker and fluorochrome used (e.g. CD4-FITC).

$\bigotimes$ The axis scales are clearly visible. Include numbers along axes only for bottom left plot of group (a 'group' is an analysis of identical markers).

\All plots are contour plots with outliers or pseudocolor plots.

$\bigotimes$ A numerical value for number of cells or percentage (with statistics) is provided.

\section{Methodology}

Sample preparation

Instrument

Software

Cell population abundance

Gating strategy
Blood collected into EDTA tubes was washed in PBS before staining with LiveDead stain according to the manufacturer's instructions. Cells were incubated with mouse serum for 10 minutes at $4^{\circ} \mathrm{C}$ to saturate the Fc receptor, and were then washed, resuspended in the appropriate antibody cocktail and incubated for 30 minutes at $4^{\circ} \mathrm{C}$. Red blood cells were lysed in Optilyse $\mathrm{C}$ Solution (Beckman Coulter), according to the manufacturer's instructions. Cells were fixed in Cell Fix solution (BD), according to the manufacturer's instructions.

Becton Dickinson LSR Fortessa X20

The FCS3.0 files obtained were exported from BD FACSDiva software and imported into FlowJo v.10.5.2 (BD Biosciences). Automated compensation was calculated with FACSDiva software and single-stained compensation beads. This compensation matrix was analyzed in detail in FlowJo, by investigating the $\mathrm{N}$-by-N view feature and the pairwise expression of all proteins stained in this study. Fluorescence minus one (FMO) experiments were run before this study, to facilitate optimization of the compensation matrix. We then adjusted the compensation matrix where necessary due to over- or under-compensation by the automatic algorithm.

Tick this box to confirm that a figure exemplifying the gating strategy is provided in the Supplementary Information.

\section{Magnetic resonance imaging}

\section{Experimental design}

Design type

Design specifications

Behavioral performance measures

Indicate task or resting state; event-related or block design.

Specify the number of blocks, trials or experimental units per session and/or subject, and specify the length of each trial or block (if trials are blocked) and interval between trials.

State number and/or type of variables recorded (e.g. correct button press, response time) and what statistics were used to establish that the subjects were performing the task as expected (e.g. mean, range, and/or standard deviation across subjects). 
Acquisition

Imaging type(s)

Specify: functional, structural, diffusion, perfusion.

Field strength

Specify in Tes/a

Sequence \& imaging parameters

Specify the pulse sequence type (gradient echo, spin echo, etc.), imaging type (EPI, spiral, etc.), field of view, matrix size, slice thickness, orientation and TE/TR/flip angle.

Area of acquisition

State whether a whole brain scan was used OR define the area of acquisition, describing how the region was determined.

Diffusion MRI

Used

Not used

Preprocessing

Preprocessing software

Provide detail on software version and revision number and on specific parameters (model/functions, brain extraction, segmentation, smoothing kernel size, etc.).

Normalization

If data were normalized/standardized, describe the approach(es): specify linear or non-linear and define image types used for transformation OR indicate that data were not normalized and explain rationale for lack of normalization.

Normalization template

Describe the template used for normalization/transformation, specifying subject space or group standardized space (e.g. original Talairach, MNI305, ICBM152) OR indicate that the data were not normalized.

Noise and artifact removal

Describe your procedure(s) for artifact and structured noise removal, specifying motion parameters, tissue signals and physiological signals (heart rate, respiration).

Volume censoring

Define your software and/or method and criteria for volume censoring, and state the extent of such censoring.

Statistical modeling \& inference

Model type and settings

Specify type (mass univariate, multivariate, RSA, predictive, etc.) and describe essential details of the model at the first and second levels (e.g. fixed, random or mixed effects; drift or auto-correlation).

Effect(s) tested

Define precise effect in terms of the task or stimulus conditions instead of psychological concepts and indicate whether ANOVA or factorial designs were used.

Specify type of analysis: $\square$ Whole brain $\square$ ROl-based $\square$ Both

Statistic type for inference

(See Eklund et al. 2016

Specify voxel-wise or cluster-wise and report all relevant parameters for cluster-wise methods.

\section{Correction}

Models \& analysis

$\mathrm{n} / \mathrm{a} \mid$ Involved in the study

$\square$ Functional and/or effective connectivity

$\square$ Graph analysis

$\square$ Multivariate modeling or predictive analysis

Functional and/or effective connectivity

Graph analysis

Report the dependent variable and connectivity measure, specifying weighted graph or binarized graph subject-or group-level, and the global and/or node summaries used (e.g. clustering coefficient, efficiency, etc.).

Multivariate modeling and predictive analysis

Specify independent variables, features extraction and dimension reduction, model, training and evaluation metrics. 